\title{
Asymptotic receptivity analysis and the Parabolized Stability Equation : a combined approach to boundary layer transition
}

\author{
By M. R. TURNER AND P. W. HAMMERTON \\ School of Mathematics, University of East Anglia, Norwich, NR4 7TJ, England, UK
}

(Received 3 July 2006)

We consider the interaction of free-stream disturbances with the leading edge of a body and its effect on the transition point. We present a method which combines an asymptotic receptivity approach, and a numerical method which marches through the Orr-Sommerfeld region. The asymptotic receptivity analysis produces a three deck eigensolution which in its far downstream limiting form, produces an upstream boundary condition for our numerical Parabolized Stability Equation (PSE). We discuss the advantages of this method against existing numerical and asymptotic analysis and present results which justifies this method for the case of a semi-infinite flat plate, where asymptotic results exist in the Orr-Sommerfeld region. We also discuss the limitations of the PSE and comment on the validity of the upstream boundary conditions. Good agreement is found between the present results and the numerical results of Haddad \& Corke (1998).

\section{Introduction}

For a body placed in a mean flow, subject to small amplitude unsteady perturbations, predicting the position of boundary layer transition depends both on the stability characteristics of the flow and the interaction of the unsteady disturbances with the boundary layer, a process known as receptivity (Morkovin 1969). Restricting attention to high Reynolds number, two-dimensional flows, the transfer of energy from the free-stream disturbance to the instability wave generally comes about through non-parallel mean flow effects, which may arise either close to the leading edge, or further downstream in localized regions of rapid streamwise variation. Once this 'seeding' of instability waves has occurred, the boundary layer disturbance evolves with distance downstream, typically decaying until the lower branch neutral stability point is reached, after which the disturbance grows until amplitudes are sufficiently high that non-linear effects lead to transition. A comprehensive review of asymptotic, numerical and experimental approaches to receptivity and transition is provided by Saric et al. (2002).

Analysis of the growth rate of boundary layer disturbances has traditionally been undertaken based on Orr-Sommerfeld theory. The main problem with this approach is that it neglects the weak streamwise growth of the boundary-layer. Non-parallel effects can be incorporated, but not rigorously in an asymptotic sense (Gaster 1974 and Saric \& Nayfeh 1975).

Bertolotti et al. (1992) present a method which incorporates non-parallel effects into a set of differential equations collectively known as the Parabolized Stability Equation (PSE). The parabolizing procedure eliminates the most dangerous upstream propagating eigenmode, and the resulting PSE can be solved by a marching procedure as long as a 
large enough step size is chosen (Andersson et al. 1998). The numerical procedure for the PSE greatly reduces computational time compared to direct numerical simulation (DNS) calculations. Compared with non-parallel Orr-Sommerfeld theory, the main advantage of the PSE is that nonlinear effects can be included by considering the non-linear form of the PSE (Bertolotti et al. 1992). In deriving the PSE, the streamwise variation of the mean flow is assumed to be slow compared to the rapid streamwise change within the boundary-layer near the receptivity region, and so becomes invalid in regions associated with boundary-layer receptivity. Since the equations have been parabolized, an upstream boundary condition, sometimes referred to as an initial condition, is required. Previous papers which consider the PSE (Bertolotti et al. 1992 and Herbert 1993) use approximations such as parallel Orr-Sommerfeld theory, or a local solution to the PSE as initial upstream conditions. However, such an approach does not take account of the amplitude of the unsteady disturbance at this point forced by the free-stream disturbances.

Receptivity problems differ from classical stability theory in that they lead to a boundary value problem rather than an eigenvalue problem, which is the result of stability theory. Receptivity analysis provides the link between free-stream forcing (be it acoustic or vortical), and the amplitude of the boundary layer disturbance. Asymptotic analysis of various receptivity regimes is available. Goldstein (1983) considered the interaction of an acoustic wave with the leading edge of a flat plate, while Heinrich \& Kerschen (1989) obtained corresponding results for a vortical disturbance. The analysis for acoustic waves was extended to include the effect of rounded leading edges (Hammerton \& Kerschen 1996), and also the effect of mean aerodynamic loading (Hammerton \& Kerschen 2005). Receptivity also arises further downstream in localized regions of rapid streamwise variation such as the vicinity of surface roughness (Goldstein 1985, Bodonyi et al. 1989 and Kerschen et al. 1990), marginal separation (Goldstein et al. 1992) or changes in surface curvature (Goldstein \& Hultgren 1987). Asymptotic analysis of these localized receptivity regions is also supplemented by a numerical 'finite-Reynolds-number approach' (Choudhari \& Streett 1992 and Crouch 1992). In each case the disturbance downstream of the receptivity region is obtained in the form of an eigensolution, independent of the nature of the free-stream forcing, together with a 'receptivity coefficient' multiplying the eigensolution. Hence comparison of the receptivity coefficients for a specific body geometry, but for different free-stream disturbances, provides a direct comparison of the relative receptivity. In addition, the receptivity coefficient is independent of Reynolds number (in the large Reynolds number limit) and these two properties make this definition of the receptivity coefficient particularly attractive. However, numerical investigations and experimental results typically focus on the amplitude of the unstable disturbance at the lower branch neutral stability point.

Numerical investigations of leading-edge receptivity can be divided into two main categories. Reed (1994) summarises DNS methods, while Fuciarelli et al. (1998) discuss such results for a flat plate with an elliptical leading edge. An alternative approach taken by Corke and co-workers is based on linearisation about the base flow, that leads to decoupling of the base and unsteady flows which can then be solved separately. Haddad \& Corke (1998) considered parabolic bodies with axis of symmetry parallel to the mean flow, Erturk \& Corke (2001) and Haddad et al. (2005) extended the analysis to parabolic bodies at an angle-of-attack to the mean flow, while Wanderley \& Corke (2001) considered bodies with elliptical leading edges in order to compare with the results of Fuciarelli et al. (1998).

Experimental results on leading-edge receptivity are summarised by Saric \& Rasmussen (1992), Saric et al. (1994) and Saric et al. (1995). Saric \& White (1998) considered receptivity on a modified super ellipse (MSE) due to free-stream sound. A modified 


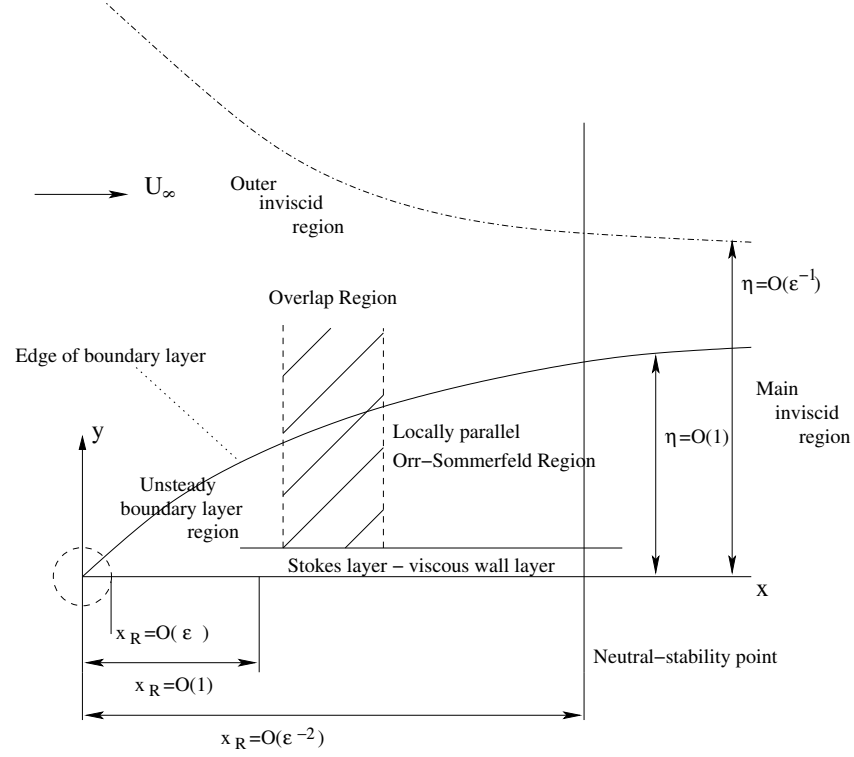

FIGURE 1. Figure of the asymptotic structure of the unsteady boundary-layer on a flat plate, showing both the leading edge region, where receptivity predicts the form of the unsteady solution far downstream, which matches on to the solution of the Orr-Sommerfeld region.

super ellipse has the leading edge directly machined onto a flat plate, which moves the pressure minimum closer to the leading edge, and removes the curvature discontinuity associated with an ellipse stuck onto a flat plate, which contributes additional receptivity. The results of Saric \& White (1998) are compared to numerical results in Wanderley \& Corke (2001).

The aim of the present paper is to show how results from receptivity theory can be combined with stability calculations in order to allow comparison with experimental measurements and numerical simulation. Here attention is restricted to leading-edge receptivity on a flat plate, though the methods can be readily extended to more general cases.

The asymptotic structure for a small amplitude unsteady disturbance interacting with a flat plate is discussed in detail by Goldstein (1983), and the boundary layer structure is illustrated in figure 1 . Here the mean flow has speed $U_{\infty}$ and the small amplitude unsteady disturbance has frequency $\omega^{*}$. Close to the leading edge, where $x_{R}=\omega^{*} x^{*} / U_{\infty}=O(1)$, the flow is governed by the unsteady boundary-layer equation. Receptivity analysis predicts the form of the unsteady disturbance far downstream in this region, and through the receptivity coefficient, the dependence of this amplitude on the free-stream forcing. When $x_{R}=O\left(\epsilon^{-2}\right)$, where $\epsilon=\left(\nu \omega^{*} / U_{\infty}^{2}\right)^{\frac{1}{6}} \ll 1$, the linearised unsteady boundary layer equation (LUBLE) solution breaks-down but can be asymptotically matched, in some intermediate region, to solutions of the classical large-Reynolds-number, small wave-number approximation to the Orr-Sommerfeld equation. For the flat-plate case, asymptotic solutions in the Orr-Sommerfeld region are available, though the analysis to the required order is particularly difficult (Goldstein 1982). Thus matching of the receptivity analysis to the asymptotic Orr-Sommerfeld solutions provides the disturbance amplitude at the lower-branch neutral stability point. However, due to the difficulty in extending this method to more general bodies, we consider using receptivity solutions as 
initial conditions for numerical solutions further downstream using the PSE formulation discussed earlier.

We derive the PSE for the case of a semi-infinite flat plate in $\S 2$, and review the key results of the asymptotic analysis of Goldstein (1983) for a flat plate in $\S 3$. In $\S 4$ we discuss the different types of initial condition for the PSE. In order to use receptivity results as an initial condition for PSE calculations, the existing receptivity analysis is extended to provide the solution for the mode shape in the outer inviscid region. Results presented in $\S 5$ show that a matching region does exist between the leading edge and Orr-Sommerfeld regions, and we discuss how this can be utilised in order to use the receptivity analysis as the initial condition to the PSE. This then provides the required link between freestream forcing in the receptivity region and the boundary layer disturbance amplitude far downstream. For small values of $\epsilon$, this method proved robust in the sense that starting PSE calculations over a range of locations provided consistent results far downstream. For moderately small values of $\epsilon$, typical of experimental studies, some problems in matching the different solutions close to the leading edge do arise. In $\S 6$ we look at ways to overcome some of these difficulties, and show how we can use the PSE method to calculate amplitudes of Tollmien-Schlichting (T-S) waves at downstream positions. We compare our results with the numerical computations of Haddad \& Corke (1998), in the limiting case of a parabola with zero nose radius. Finally we compare our results with the methods used by Wanderley \& Corke (2001) in their attempt to extrapolate numerical results, close to the neutral stability point, back to the receptivity region at the leading edge.

\section{Formulation of the Parabolized Stability Equation}

Here we briefly summarise the derivation of the PSE for the case of two-dimensional disturbances in the Blasius boundary layer. The PSE is not just restricted to twodimensional problems, and the three-dimensional version can be found in Herbert (1993)

The Cartesian coordinate system $\left(x^{*}, y^{*}\right)$ is used, where $x^{*}$ is the dimensional streamwise direction and $y^{*}$ is normal to the plate. The Navier-Stokes equations are written in terms of the stream function $\Psi$ to satisfy continuity identically,

$$
\left(\frac{\partial}{\partial t}-\frac{1}{R_{0}} \nabla^{2}+\frac{\partial \Psi}{\partial y} \frac{\partial}{\partial x}-\frac{\partial \Psi}{\partial x} \frac{\partial}{\partial y}\right) \nabla^{2} \Psi=0
$$

where

$$
R_{0}=\frac{U_{\infty} \delta_{0}}{\nu}
$$

and all quantities are non-dimensionalized using the velocity $U_{\infty}$ and the fixed length $\delta_{0}=\left(\nu x_{0}^{*} / U_{\infty}\right)^{\frac{1}{2}}$. Here $\nu$ is the kinematic viscosity and $x_{0}^{*}$ is the dimensional distance from the leading edge at which the analysis is started. The corresponding non-dimensional distance is given by $x_{0}=R_{0} . R_{0}$ is the Reynolds number based on $\delta_{0}$, and is assumed to be large, so that the flow field is inviscid and irrotational everywhere except in the vicinity of the flat plate's surface.

The stream function is split into a disturbance part $\psi(x, y, t)$ and a steady base flow $\Psi_{B}(x, y)=f(\eta)\left(x / R_{0}\right)^{\frac{1}{2}}+O\left(R_{0}^{-2}\right)$, where $f$ is the Blasius function, and satisfies

$$
f^{\prime \prime \prime}+f f^{\prime \prime}=0, \quad f(0)=f^{\prime}(0)=0 \quad f^{\prime} \longrightarrow 1 \quad \text { as } \quad \eta \longrightarrow \infty,
$$

with $\eta=R_{0}^{\frac{1}{2}} y /(2 x)^{\frac{1}{2}}$.

The equation for $\psi$ is obtained by substituting the combined flow into (2.1) and subtracting off the equation satisfied by the mean flow (Bertolotti et al. 1992). Then a 
solution for $\psi$ is sought in the form of a spatially evolving two-dimensional wave of constant frequency $\omega$, with a local streamwise wavenumber $\alpha(x)$, and a complex mode shape $\phi(x, y)$, of the form

$$
\psi(x, y, t)=\phi(x, y) \exp (i(\theta(x)-\omega t))+\text { complex conjugate }
$$

where

$$
\frac{d \theta}{d x}=\alpha(x)
$$

The disturbance amplitude is assumed to be sufficiently small, $|\psi| \ll 1$, so that the non-linear terms in $\psi$ can be neglected. Non-linear terms are neglected, not just for simplicity, but also because we are only interested in running calculations up to, and slightly beyond the lower branch neutral stability point. The non-linear effects usually only become significant as the wave amplitude grows, which happens downstream of this point. Bertolotti et al. (1992) also discussed a non-linear version of the PSE, which can be used to incorporate non-linear effects, which would help to take calculations up to upper branch.

The key assumption in the PSE formulation is that the variation of $\alpha$ and $\phi$ is sufficiently small so that $\partial^{2} \alpha / \partial x^{2}$ and $\partial^{2} \phi / \partial x^{2}$ and products of first derivatives $\partial \alpha / \partial x$, $\partial \phi / \partial x$ are negligible. This assumption has been observed to hold for T-S waves both in experiments and in numerical computations (Morkovin 1985). In addition, the neglection of these terms can be argued by noting that for a flat plate the streamwise variation of the mean flow $\Psi_{B}$ varies on a length scale of $O\left(R_{0}^{-1}\right)$, suggesting that the streamwise variation of $\phi$ and $\alpha$ is at most $O\left(R_{0}^{-1}\right)$ and that the magnitude of $\phi_{x x}, \alpha_{x x}$ and $\phi_{x} \alpha_{x}$ can be at most $O\left(R_{0}^{-2}\right)$. Thus these terms can be neglected if we neglect all terms of $O\left(R_{0}^{-2}\right)$ in our governing equation.

These assumptions lead to the derivation of the linear form of the PSE, which written in operator form is

$$
\left(L_{0}+L_{1}\right) \phi+M \frac{\partial \phi}{\partial x}+\frac{d \alpha}{d x} N \phi=0
$$

where

$$
\begin{aligned}
L_{0} & =-\frac{1}{R_{0}}\left(D^{2}-\alpha^{2}\right)^{2}+\left(i \alpha \frac{\partial \Psi_{B}}{\partial y}-i \omega\right)\left(D^{2}-\alpha^{2}\right)-i \alpha \frac{\partial^{3} \Psi_{B}}{\partial y^{3}} \\
L_{1} & =\frac{\partial^{3} \Psi_{B}}{\partial x \partial y^{2}} D-\frac{\partial \Psi_{B}}{\partial x}\left(D^{2}-\alpha^{2}\right) D \\
M & =\frac{\partial \Psi_{B}}{\partial y}\left(D^{2}-3 \alpha^{2}\right)+2 \alpha \omega-\frac{\partial^{3} \Psi_{B}}{\partial y^{3}} \\
N & =\omega-3 \alpha \frac{\partial \Psi_{B}}{\partial y}
\end{aligned}
$$

and $D \equiv d / d y$.

Equation (2.5) is very similar to the one derived by Bertolotti et al. (1992), except we have neglected the $O\left(R_{0}^{-1}\right)$ terms from operators $M$ and $N$, as these only contribute to the solution at $O\left(R_{0}^{-2}\right)$, so in our opinion the approximation is more consistent, in the sense that we have neglected all the $O\left(R_{0}^{-2}\right)$ terms rather than neglecting most of them but retaining two terms, as in Bertolotti et al. (1992). This approach has been adopted by Andersson et al. (1998), and our findings have shown that these $O\left(R_{0}^{-2}\right)$ terms have no significant effect on the solution to the problem.

A second equation is required to resolve the ambiguity of the partition (2.4) into two functions of $x$. A normalization condition is imposed on $\phi$ which restricts rapid variation 
in the $x$ direction. To achieve this, the normalization condition is defined as

$$
\int_{0}^{\infty} \phi_{x} \phi^{\dagger} d y=0,
$$

where $\nmid$ denotes the complex conjugate. This condition minimises the streamwise change $\partial \phi / \partial x$ in a weighted sense across the $y$ domain, and hence puts the majority of the streamwise variation with $x$ into the wavenumber $\alpha$. There are many other possible normalization conditions, all of which would lead to the same total growth rate used in $\S 5$, however we chose (2.10) as this was the choice used by other authors (Herbert 1993 and Andersson et al. 1998), and was relatively easy to implement numerically.

The approach taken in the present implementation of the PSE scheme, in contrast to previous work, is that $\eta$, as defined following (2.3), is used as the wall normal coordinate rather that $y$. Hence the boundary layer growth downstream is naturally accounted for in the numerical mesh. The semi-infinite domain $\eta \in[0, \infty)$ is mapped to the domain $\bar{\eta} \in[-1,1]$ by

$$
\bar{\eta}=\frac{\eta-L}{\eta+L},
$$

where $L$ denotes a constant map parameter, chosen to match the width of the function to be expanded. In this problem, the main variation of the shape function occurs for $\eta<20$, hence choosing $L=20$ is a reasonable value, and we found these results agree with those of the case $L=45$, so there is some amount of flexibility in this value.

The numerical procedure consists of expanding the shape function, $\phi$, as a sum of Chebyshev polynomials. The system is then solved at each streamwise position for the coefficients multiplying each polynomial, with iterations on $\phi$ and the wavenumber, $\alpha$, until the normalization condition (2.10) is satisfied. This numerical method is then equivalent to the one used by Bertolotti et al. (1992), and more details are contained in this paper.

For the purposes of this paper, we are only interested in the propagation of the eigensolutions from the leading edge region, hence we solve (2.5) with homogeneous boundary conditions, and as an upstream boundary condition, we stipulate

$$
\phi\left(x_{0}, y\right)=\hat{F}(y), \quad \alpha\left(x_{0}\right)=\alpha_{0},
$$

where $x_{0}$ is the dimensionless starting position on the plate. These conditions are determined by the mean boundary layer at $x_{0}$ and by the interaction of the unsteady free-stream disturbance with the boundary-layer upstream of $x_{0}$.

\section{Asymptotic analysis for a flat plate}

\subsection{Leading edge receptivity analysis}

Close to the leading edge of the flat plate, the PSE is not valid, because we have rapid growth of the boundary layer, and the assumption that $\alpha_{x x}, \phi_{x x}$ and $\alpha_{x} \phi_{x}$ are small is no longer valid. This region occurs when $x_{R}=O(1)$, where $x_{R}$ is equivalent to the streamwise variable $x$ used by Goldstein (1983). Hence we have a different balance of terms at leading order. The solution in this receptivity region is determined in the form of a three deck solution as shown in figure 1. The Stokes layer close to the wall is the deck in which viscous terms are most important, and the solution in this deck satisfies the no slip condition at the plate's surface. The outer inviscid region lies outside the boundary layer, and in this deck the disturbance amplitude tends to zero as $\eta \longrightarrow \infty$. 
Between these two decks there is the main inviscid region, and the solution in this deck must match onto the other two decks.

The governing equation in this region for a flat plate in a uniform flow of speed $U_{\infty}$ plus a small amplitude harmonic perturbation of the dimensional frequency $\omega^{*}$ takes the form

$$
-i \tilde{\nabla}^{2} \psi+x_{R}^{\frac{1}{2}}\left[\frac{\partial\left(x_{R}^{-1} \tilde{\nabla}^{2} \psi, x_{R}^{\frac{1}{2}} f\right)}{\partial\left(x_{R}, \eta\right)}+\frac{\partial\left(x_{R}^{-\frac{1}{2}} f^{\prime \prime}, \psi\right)}{\partial\left(x_{R}, \eta\right)}\right]=\tilde{\nabla}^{2}\left(\frac{1}{2 x_{R}} \tilde{\nabla}^{2} \psi\right),
$$

where

$$
\tilde{\nabla}^{2}=\frac{\partial^{2}}{\partial \eta^{2}}+2 \epsilon^{6} x_{R} \frac{\partial^{2}}{\partial x_{R}^{2}}+\epsilon^{6} \frac{\partial}{\partial x_{R}}, \quad \epsilon=R e^{-\frac{1}{6}}=F^{\frac{1}{6}}=\left(\frac{U_{\infty}^{2}}{\nu \omega^{*}}\right)^{-\frac{1}{6}},
$$

with conditions on the wall $\psi(0)=\psi_{\eta}(0)=0$ and $\psi$ matching to the inviscid solution for large $\eta$. In (3.1), correction terms, which remain uniformly small in all the regimes we considered, have been dropped. The value $R e$ is defined as the Reynolds number based on the acoustic length scale $U_{\infty} / \omega^{*}$, and $F=\omega^{*} \nu / U_{\infty}$ is the dimensionless frequency, commonly used in stability calculations.

We seek a solution of (3.1), in the limit $\epsilon \longrightarrow 0$ with $x_{R}=O(1)$, of the form $\psi=$ $\psi_{0}\left(x_{R}, \eta\right)+O\left(\epsilon^{6}\right)$ where $\psi_{0}$ satisfies

$$
\left(-i+f^{\prime} \frac{\partial}{\partial x_{R}}\right) \psi_{0 \eta}-f^{\prime \prime} \psi_{0 x_{R}}-\frac{1}{2 x_{R}}\left(f \psi_{0 \eta}\right)_{\eta}-\frac{1}{2 x_{R}} \psi_{0 \eta \eta \eta}=h\left(x_{R}\right),
$$

and $h\left(x_{R}\right)$ is determined by the unsteady forcing of the boundary layer by the free-stream disturbance. This equation is known as the linearised unsteady boundary layer equation (LUBLE).

Far downstream in this region, the solution for $\psi_{0}$ consists of a combination of a Stokes solution, and a sum of eigensolutions satisfying homogeneous boundary conditions (Lam \& Rott 1960 \& 1993; Brown \& Stewartson 1973). The two sets of eigensolutions differ fundamentally and their precise relationship is unclear (Hammerton 1999). The importance of the Lam-Rott eigensolutions in receptivity analysis is that they exhibit wavelength shortening and hence provide the link between long wavelength free-stream disturbances and much shorter scale instability waves in the boundary layer. The $n^{\text {th }}$ Lam-Rott asymptotic eigensolution takes the form,

$$
\psi_{0}^{(n)}=C_{n} x_{R}^{\tau_{n}} g_{0}\left(x_{R}, \eta\right) \exp \left(-\frac{e^{-\frac{7 \pi i}{4}}\left(2 x_{R}\right)^{\frac{3}{2}}}{3 U_{0}^{\prime} \zeta_{n}^{\frac{3}{2}}}\right) \quad \text { as } \quad x_{R} \longrightarrow \infty,
$$

where $C_{n}$ is an arbitrary constant, $U_{0}^{\prime}=f^{\prime \prime}(0)=0.4696, \tau_{n}$ is expressed in terms of integrals of Airy functions involving $\zeta_{n}$ (Hammerton \& Kerschen 1996), and $\zeta_{n}$ is the $n^{\text {th }}$ root of $A i^{\prime}\left(\zeta_{n}\right)=0$ (where $A i^{\prime}$ denotes the derivative of the Airy function). For the transition problem considered here, the first eigensolution is of particular interest, in which case $\tau_{1}=-0.6921$ and $\zeta_{1}=-1.0188$.

Note that the wavelength of (3.4) is proportional to $x_{R}^{-\frac{1}{2}}$, hence when $x_{R}$ gets large, (3.3) is no longer valid as the leading order balance of (3.1) due to terms in $\partial / \partial x_{R}$ becoming large. This is found to occur when $x_{R}=O\left(\epsilon^{-2}\right)$, so we have to consider a different balance of terms in this region.

\subsection{Asymptotic Orr-Sommerfeld analysis}

The region where $x_{R}=O\left(\epsilon^{-2}\right)$ is known as the Orr-Sommerfeld region, and we define a scaled, $O(1)$, variable in this region by $\tilde{x}_{1}=2 \epsilon^{2} x_{R} / U_{0}^{\prime 2}$. 
We seek a solution to the governing equation in this region in the form

$$
\psi_{0}=\gamma\left(\tilde{x}_{1}, \eta\right) A\left(\tilde{x}_{1}\right) \exp \left(\frac{i U_{0}^{\prime 2}}{2 \epsilon^{3}} \int_{0}^{\tilde{x}_{1}} \kappa d \tilde{x}_{1}\right),
$$

where $A\left(\tilde{x}_{1}\right)$ is a slowly varying function of $\tilde{x}_{1}$ and $\gamma$ is the mode shape. We define the growth rate of the disturbance by the real part of

$$
\frac{1}{\psi_{0}} \frac{\partial \psi_{0}}{\partial \tilde{x}_{1}}=\frac{\gamma_{\tilde{x}_{1}}}{\gamma}+\frac{A_{\tilde{x}_{1}}}{A}+\frac{i U_{0}^{\prime 2}}{2 \epsilon^{3}} \kappa .
$$

In the Orr-Sommerfeld region the wavenumber can be expanded in the form

$$
\kappa\left(\tilde{x}_{1}\right)=\kappa_{0}\left(\tilde{x}_{1}\right)+\epsilon \kappa_{1}\left(\tilde{x}_{1}\right)+\epsilon^{2} \kappa_{2}\left(\tilde{x}_{1}\right)+\epsilon^{3}(\ln \epsilon) \kappa_{3}\left(\tilde{x}_{1}\right)+O\left(\epsilon^{3}\right),
$$

where the terms $\kappa_{0}, \kappa_{1}, \kappa_{2}$, and $\kappa_{3}$ are given in Goldstein (1983). Here we give expressions only for the first two terms. Defining

$$
\zeta_{00}=e^{-\frac{5}{6} i \pi}\left(\frac{\tilde{x}_{1}^{\frac{1}{2}}}{\kappa_{0}}\right)^{\frac{2}{3}}, \quad H\left(\zeta_{00}\right)=\frac{e^{\frac{5}{2} i \pi} \zeta_{0}^{2} A i^{\prime}\left(\zeta_{00}\right)}{\int_{\infty_{1}}^{\zeta_{00}} A i(\zeta) d \zeta}
$$

$\kappa_{0}$ and $\kappa_{1}$ are determined by

$$
\begin{aligned}
H\left(\zeta_{00}\right) & =\tilde{x}_{1}^{\frac{3}{2}}, \\
\frac{\kappa_{1}}{\kappa_{0}} & =-\frac{3}{2} e^{\frac{1}{4} i \pi} \zeta_{00}^{\frac{1}{2}} \tilde{x}_{1}\left(2-\frac{\tilde{x}_{1}^{\frac{3}{2}} J_{1}}{i \zeta_{00}^{3}}\right) / H^{\prime}\left(\zeta_{00}\right) .
\end{aligned}
$$

The equation for $\zeta_{00}$ gives a set of solutions and it can be shown that as $\tilde{x}_{1} \longrightarrow 0$, the solution corresponding to the $n^{\text {th }}$ root matches back to the $n^{\text {th }}$ Lam-Rott eigensolution (3.4). The growth rate obtained using the first root of $\zeta_{00}$ becomes positive further downstream and it is for this reason that the first Lam-Rott eigensolution is of interest. The numerical coefficient $C_{1}$ which is determined by the receptivity analysis then gives the amplitude scaling of the mode which becomes unstable and hence is termed the receptivity coefficient.

From (3.5) it is seen that the $O\left(\epsilon^{3}\right)$ term in $\kappa$ enters the analysis at the same order as $A\left(\tilde{x}_{1}\right)$ in determining the algebraic correction to the growth rate of the disturbance. The analysis at this order is very complicated and was not presented in Goldstein (1983), though results were obtained in an earlier paper (Goldstein 1982).

When analysing the full transition problem, combining receptivity and stability processes, inclusion of algebraic growth terms is clearly important, especially if the parameter $\epsilon$ is only moderately small. The complexity of the analysis at this order even for a flat plate, makes prediction of disturbance amplitudes at lower branch difficult. For finite thickness bodies, with a rounded leading edge tending to a flat plate far downstream, we find that the geometry enters the the problem for $\kappa$ via the large $x_{R}$ expansion for the base flow at $O\left(\ln \left(x_{R}\right) / x_{R}\right)$, and via the term $A\left(\tilde{x}_{1}\right)$ at $O(1)$. Hence for moderate values of $\epsilon$, and small $x_{R}$, the accurate evaluation of the $A\left(\tilde{x}_{1}\right)$ term becomes important. We find however that at this order, such analysis is impractical. For this reason we choose to consider numerical solutions in the Orr-Sommerfeld region using PSE methods.

\section{Upstream boundary conditions for the PSE}

To march the PSE solution downstream, we need to stipulate an upstream boundary condition (2.12). This next section compares three possible choices for this. 


\subsection{Local solution to the PSE}

Possibly the easiest and most convenient upstream boundary condition to the PSE is to assume that at the starting point the boundary layer is parallel, hence streamwise derivatives of $\phi, \alpha$ and $\Psi_{B}$ are all zero, and the problem reduces to the Orr-Sommerfeld problem

$$
L_{0} \phi=\left(-\frac{1}{R_{0}}\left(D^{2}-\alpha^{2}\right)^{2}+\left(i \alpha \frac{\partial \Psi_{B}}{\partial y}-i \omega\right)\left(D^{2}-\alpha^{2}\right)-i \alpha \frac{\partial^{3} \Psi_{B}}{\partial y^{3}}\right) \phi=0 .
$$

The initial conditions come from solving this eigenvalue problem for the most unstable eigenvalue $\alpha$ and corresponding eigenfunction $\phi$ at the starting value of $x$.

To use the Orr-Sommerfeld approximation above, we have to be far enough from the leading edge of the body so that the growth of the boundary layer is very small. However, in order to consider the effect of leading edge receptivity on transition, we would like to start our PSE calculations from closer to the leading edge, where there is a small, but significant, change in boundary layer thickness.

An improved method, taking some account of non-parallel effects, is described by Bertolotti et al. (1992) where the solution to (2.5) is found locally about some value $x_{0}$. We expand the local solution as a Taylor series in powers of $\xi=x-x_{0}$ and note that the second derivatives of $\phi$ and $\alpha$ with respect to $x$ can be neglected because of our assumption that these are at most $O\left(R_{0}^{-2}\right)$.

The Taylor series gives $\phi(x, y)=\phi_{0}+\xi \phi_{1}$ and $\alpha(x)=\alpha_{0}+\xi \alpha_{1}$, which on introduction into (2.5) and requiring that the equation be valid for varying $\xi$ produces two equations,

$$
\begin{array}{r}
\left(L_{0}+L_{1}+\alpha_{1} N\right) \phi_{0}+M \phi_{1}=0 \\
\left(L_{3}+i \alpha_{1} M\right) \phi_{0}+L_{0} \phi_{1}=0,
\end{array}
$$

where

$$
L_{3}=i \alpha_{0} \frac{\partial^{2} \Psi_{B}}{\partial x \partial y}\left(D^{2}-\alpha_{0}^{2}\right)-i \alpha_{0} \frac{\partial^{4} \Psi_{B}}{\partial x \partial y^{3}},
$$

and in $L_{0}, L_{1}, M$ and $N$, defined in (2.6-2.9), $\alpha$ is replaced by $\alpha_{0}$.

To solve this problem, we consider the approximation $\alpha=$ constant, i.e. $\alpha_{1}=0$, which is effectively a different normalization condition to (2.10). This approach leads to us solving a simple linear system problem, using methods devised by Bridges \& Morris (1984).

The eigenvalue problem becomes

$$
\left[\begin{array}{cc}
L_{0}+L_{1} & M \\
L_{3} & L_{0}
\end{array}\right]\left[\begin{array}{l}
\phi_{0} \\
\phi_{1}
\end{array}\right]=\left[\begin{array}{l}
0 \\
0
\end{array}\right]
$$

which at $x=x_{0}$ gives us the upstream boundary condition $\alpha=\alpha_{0}$ and $\phi=\phi_{0}$. Bertolotti et al. (1992) shows that (4.4) produces a pair of eigenvalues which approach the eigenvalue of the Orr-Sommerfeld problem as $R_{0} \longrightarrow \infty$. Because of this reason it becomes ambiguous as to which eigenvalue we take as our initial condition, which we shall address in the results section. Bertolotti et al. (1992) suggests other iterative methods to solve (4.2) and (4.3), but his analysis shows that there is no advantage in using these methods compared to the one above.

With both the Orr-Sommerfeld and local PSE conditions, we lack information about the initial amplitude of the eigensolution. This leaves the final amplitude, after the PSE calculation, as a multiple of the initial amplitude, which is unknown. The reason for this, is that the problem in the Orr-Sommerfeld region is an eigenvalue problem, and hence contains no information from the leading edge, or from the free-stream disturbance. 


\subsection{Leading edge asymptotics}

Leading edge receptivity analysis supplies us with an initial amplitude for the eigensolution, since far downstream we know the complete solution, given by (3.4), up to the arbitrary constant $C_{n}$, which in turn can be determined by the methods described in Goldstein et al. (1983).

However, we cannot use the form of $g_{0}$ given in (3.4), because it is only valid up to $\eta=O(1)$, whereas the PSE requires a boundary condition defined for $\eta \in[0, \infty)$. Thus the LUBLE solution in the outer inviscid region, with $g_{0} \longrightarrow 0$ as $\eta \longrightarrow \infty$ is required. This outer inviscid solution is not given in Goldstein (1983) and is derived here.

To analyse the outer inviscid solution, we consider the following scaled variables

$$
x_{R}=\epsilon^{-l} X \quad \eta=\epsilon^{-m} \quad l, m>0 .
$$

As the outer inviscid solution occurs in the region $\eta \longrightarrow \infty$, we can approximate the Blasius function by

$$
f(\eta) \sim \eta-\tilde{c}_{1}+\text { exponentially small terms, }
$$

where $\tilde{c}_{1}=1.21678$. In terms of the new variables, the operator $\tilde{\nabla}^{2}$ appearing in $(3.2)$ becomes

$$
\tilde{\nabla}^{2}=\epsilon^{2 m} \frac{\partial^{2}}{\partial N^{2}}+2 \epsilon^{6+l} X \frac{\partial^{2}}{\partial X^{2}}+\epsilon^{6+l} \frac{\partial}{\partial X} .
$$

Motivated by the form of the solution in the main layer (3.4), we assume $\psi$ is of the form

$$
\psi=P(X, N) \exp \left(-\left(\frac{2^{\frac{3}{2}} \lambda}{3 U_{0}^{\prime}}\right) \epsilon^{-\frac{3}{2} l} X^{\frac{3}{2}}\right),
$$

where $P(X, N)$ is a function to be found and $\lambda=e^{-\frac{7 \pi i}{4}} / \zeta_{n}^{\frac{3}{2}}$. Substituting into (3.1) and using (4.5) and (4.6), we find that

$$
l+m=3,
$$

in order to give a non-trivial leading order balance of terms.

To provide a suitable initial condition for a PSE solution, the LUBLE solution is required in the overlap region, corresponding to $x_{R}=O\left(\epsilon^{-2}\right)$. Which with $l=2$, and $m=1$, in our analysis leads to

$$
P_{N N}+\frac{9}{2}\left(\frac{2^{\frac{3}{2}} \lambda}{3 U_{0}^{\prime}}\right)^{2} X^{2} P+O\left(\epsilon^{3}\right)=0 .
$$

The solution of (4.7) which is bounded as $X \longrightarrow \infty$ is

$$
P=\left(B_{0}(X)+\epsilon B_{1}(X)\right) \exp \left(-\frac{2 \lambda i X N}{U_{0}^{\prime}}\right)+O\left(\epsilon^{2}\right),
$$

and matching to the main inviscid region gives

$$
P(x, \eta)=x_{R}^{\tau_{n}}\left(\frac{U_{0}^{\prime} i}{\lambda}+\left(2 x_{R}\right)^{\frac{1}{2}}\right) \exp \left(-\frac{\epsilon^{3} \lambda i\left(2 x_{R}\right) \eta}{U_{0}^{\prime}}\right)+O\left(\epsilon^{2}\right) .
$$

It can also be shown that the solution is valid closer to the leading edge where $x_{R}=$ $O\left(\epsilon^{-1}\right)$. However it is not valid at the leading edge, because here the motion is governed by the full Navier-Stokes equations.

Using $g_{0}$ and (4.8), we can represent the mode shape in the leading edge region in the 
form of a composite function, for which we take

$$
\begin{aligned}
& \text { composite } \\
& \text { solution }
\end{aligned}=\left(\begin{array}{l}
\text { inner deck } \\
\text { solution }
\end{array}+\begin{array}{l}
\text { main deck } \\
\text { solution }
\end{array}-\begin{array}{l}
\text { overlap } \\
\text { solution }
\end{array}\right) \times \begin{aligned}
& \text { outer deck } \\
& \text { exponential }
\end{aligned},
$$

$\mathrm{SO}$

$$
\begin{array}{r}
g_{0}\left(x_{R}, \eta\right)=\left(x_{R}\right)^{\tau_{n}}\left(\left(2 x_{R}\right)^{\frac{1}{2}} f^{\prime}(\eta)+U_{0}^{\prime} \frac{\int_{0}^{\sigma}(\sigma-\tilde{\sigma}) \omega(\tilde{\sigma}) d \tilde{\sigma}}{\int_{0}^{\infty} \omega(\tilde{\sigma}) d \tilde{\sigma}}-U_{0}^{\prime}\left(2 x_{R}\right)^{\frac{1}{2}} \eta\right) \\
\times \exp \left(-\frac{\epsilon^{3} i \lambda\left(2 x_{R}\right) \eta}{U_{0}^{\prime}}\right) .
\end{array}
$$

We now use this expression as the initial condition for the PSE calculations. The receptivity and PSE variables are related by

$$
x_{R}=\frac{R_{0}}{R e} x
$$

and hence if the starting point for the PSE calculation is taken to be $\tilde{x}_{1}=\tilde{x}_{1}^{(0)}$, we have

$$
x_{0}=R_{0}=\epsilon^{-4} U_{0}^{\prime}\left(\frac{\tilde{x}_{1}^{(0)}}{2}\right)^{\frac{1}{2}}, \quad \omega=\frac{R_{0}}{R e} .
$$

From (3.4) and (4.10) the initial wave number is then given by

$$
\alpha\left(x_{0}\right)=i \lambda \epsilon^{6} R_{0}\left(\tilde{x}_{1}^{(0)}\right)^{\frac{1}{2}},
$$

and the initial shape function for the PSE calculation is given by

$$
\phi\left(x_{0}, \eta\right)=C_{1} g\left(x_{R}^{(0)}, \eta\right), \quad x_{R}^{(0)}=\frac{\epsilon^{-2} U_{0}^{\prime 2} \tilde{x}_{1}^{(0)}}{2} .
$$

Thus to stipulate an initial condition we only require the value of $\epsilon$ and a choice of value for $\tilde{x}_{1}^{(0)}$.

\section{Results}

In this section, we present results which illustrates the matching region on a flat plate, and we give streamwise bounds for this region. We also demonstrate that using the results of receptivity analysis as the initial condition for the PSE is consistent with other initial conditions.

To make the comparison of the results easier, we split the amplitude function $\phi(x, \eta)$, defined in (2.4), in the following way,

$$
\phi(x, \eta)=\phi_{\max }(x) \bar{\phi}(x, \eta),
$$

where the maximum value of $\bar{\phi}$ is 1 . The stream function is then given by

$$
\psi=\bar{\phi}(x, \eta) \exp (i \tilde{\theta}(x)-\omega t)+\text { complex conjugate }
$$

with

$$
\frac{d \tilde{\theta}}{d x}=G(x)
$$

where $G(x)$ now contains all the wave amplitude information and is of the form

$$
G(x)=\frac{R e}{R_{0}}\left(i \alpha+\frac{1}{\phi_{\max }} \frac{\partial \phi_{\max }}{\partial x}\right) .
$$


(a)

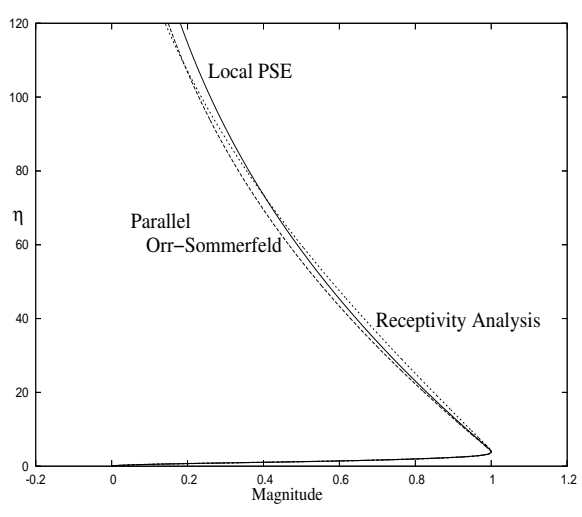

(b)

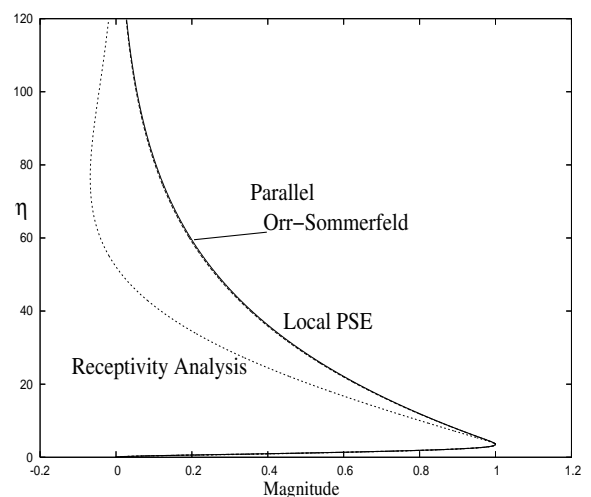

Figure 2. Comparison of the real part of the initial mode shapes of the three regimes for $\epsilon=0.1$ at streamwise locations (a) $\tilde{x}_{1}^{(0)}=0.3$, and (b) $\tilde{x}_{1}^{(0)}=1.0$.

(a)

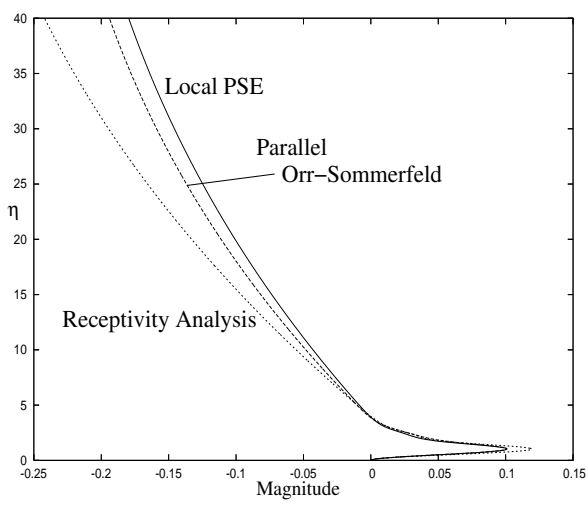

(b)

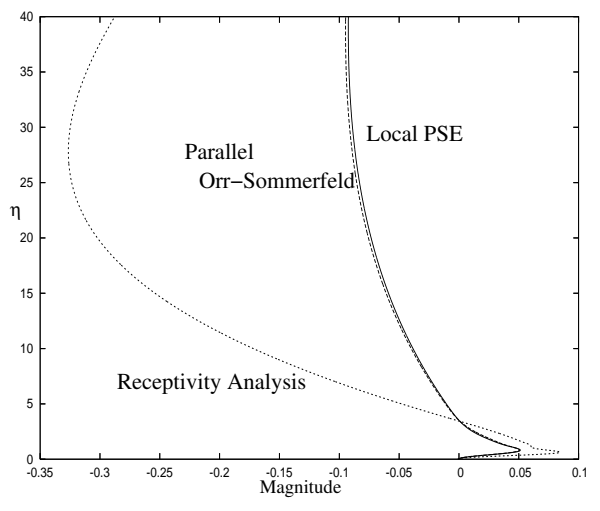

FiguRE 3. Comparison of the imaginary part of the initial mode shapes for the three regimes for $\epsilon=0.1$ at streamwise locations (a) $\tilde{x}_{1}^{(0)}=0.3$, and (b) $\tilde{x}_{1}^{(0)}=1.0$.

Although $G(x)$ is defined as a function of the PSE streamwise variable, we plot it as a function of $\tilde{x}_{1}$, for easy comparison with Goldstein's results.

In figures 2 to 5 , we compare initial mode shapes at two $\tilde{x}_{1}^{(0)}$ values and for two values of $\epsilon$. These show the existence of a matching region, in the limit $\epsilon \longrightarrow 0$, where the solutions from the Orr-Sommerfeld regime and the leading edge regime match onto one another.

Figures 2 and 3 show a comparison between the initial mode shapes of the three upstream boundary conditions at two different starting positions, $\tilde{x}_{1}^{(0)}=0.3$ and $\tilde{x}_{1}^{(0)}=1$ when $\epsilon=F^{\frac{1}{6}}=0.1$. For this value of $\epsilon, \tilde{x}_{1}^{(0)}=1$ corresponds to the downstream Reynolds number $R_{x}=U_{\infty} x^{*} / \nu=1.1 \times 10^{7}$. The real parts, figure 2 , and imaginary parts, figure 3 , compare very well close to the wall for both starting points, but as we move away from the wall, they all decay to zero at slightly different rates, and at $\tilde{x}_{1}^{(0)}=1$ the parallel Orr-Sommerfeld and local PSE mode shapes vary more from the receptivity mode shape than they do at $\tilde{x}_{1}^{(0)}=0.3$. This suggests that $\tilde{x}_{1}^{(0)}=0.3$ lies closer to the overlap region than $\tilde{x}_{1}^{(0)}=1$. Considering smaller values of $\tilde{x}_{1}^{(0)}$ for this value of $\epsilon$ does not improve the agreement between the three mode shapes since the parallel Orr-Sommerfeld equation and the local PSE become invalid as $\tilde{x}_{1}^{(0)} \longrightarrow 0$ due to non-parallel effects entering at leading order. Also as we let $\tilde{x}_{1}^{(0)} \longrightarrow 0$, we encounter problems identifying the most 
(a)
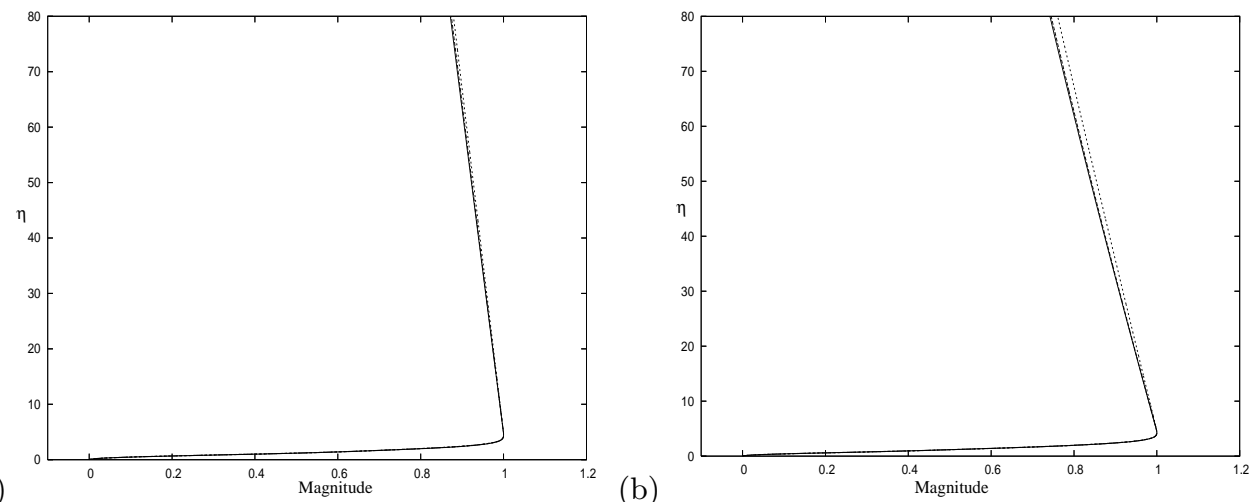

Figure 4. Comparison of the real part of the initial mode shapes for $\epsilon=0.05$ for the leading edge receptivity, parallel Orr-Sommerfeld and local PSE analysis, where the line styles correspond to those in figures 2 and 3 . In (a) $\tilde{x}_{1}^{(0)}=0.1$, the 3 mode shapes lie over each other, and in (b) $\tilde{x}_{1}^{(0)}=0.2$, only the leading edge mode shape is distinguishable from the other two.

(a)

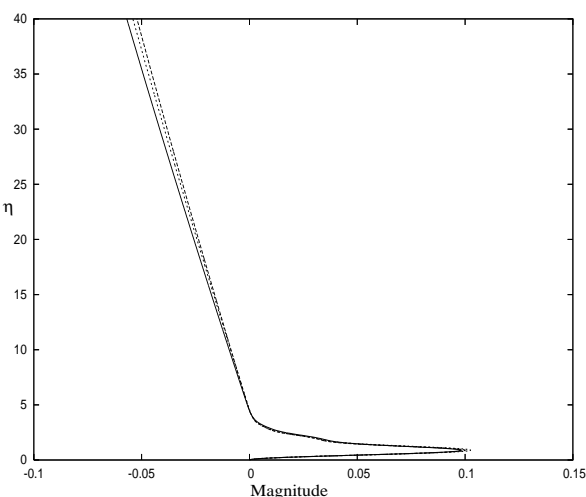

(b)

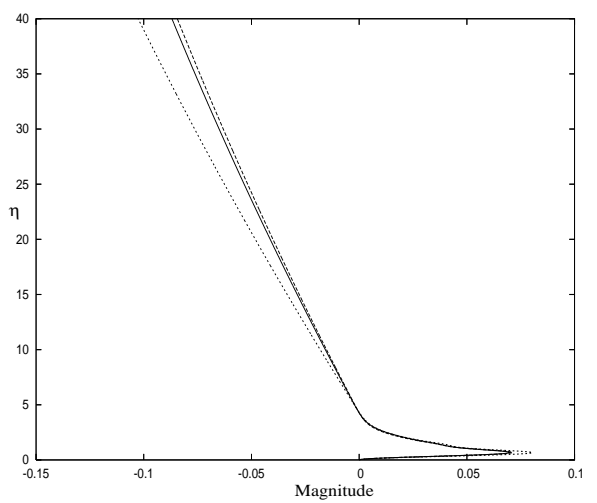

FIGURE 5. Comparison of the imaginary part of the initial mode shapes for $\epsilon=0.05$ for the leading edge receptivity, parallel Orr-Sommerfeld and local PSE analysis, where the line styles correspond to those in figures 2 and 3 . In (a) $\tilde{x}_{1}^{(0)}=0.1$, the solutions are the same for small $\eta$, and in (b) $\tilde{x}_{1}^{(0)}=0.2$, the leading edge mode shape is more distinguishable.

unstable eigenvalue for both the parallel Orr-Sommerfeld and local PSE calculations. It is found that the unstable eigenvalue becomes indistinguishable from the discrete approximation to the continuous spectrum of eigenvalues in each case.

In order to illustrate the existence of a matching region more clearly, we consider corresponding results for a smaller value of $\epsilon$. With $\epsilon=0.05$, we can solve the parallel Orr-Sommerfeld and local PSE equations closer to $\tilde{x}_{1}^{(0)}=0$, and figures 4 and 5 compare the real and imaginary parts of the mode shapes respectively at $\tilde{x}_{1}^{(0)}=0.1$ and $\tilde{x}_{1}^{(0)}=0.2$. For this smaller value of $\epsilon$, figure 4 (a) shows that the real part of the three solutions at $\tilde{x}_{1}^{(0)}=0.1$ overlap each other while figure $5(\mathrm{a})$ shows that for the imaginary part of the solution at this point, the local PSE is in fact in slightly better agreement with the receptivity solution than the parallel Orr-Sommerfeld solution. From these figures it is clear that $\tilde{x}_{1}^{(0)}=0.1$ lies within an overlap region between the leading edge region and the Orr-Sommerfeld region.

Figure 6 shows a comparison of the real part of the growth rate, $G$, calculated using 
(a)

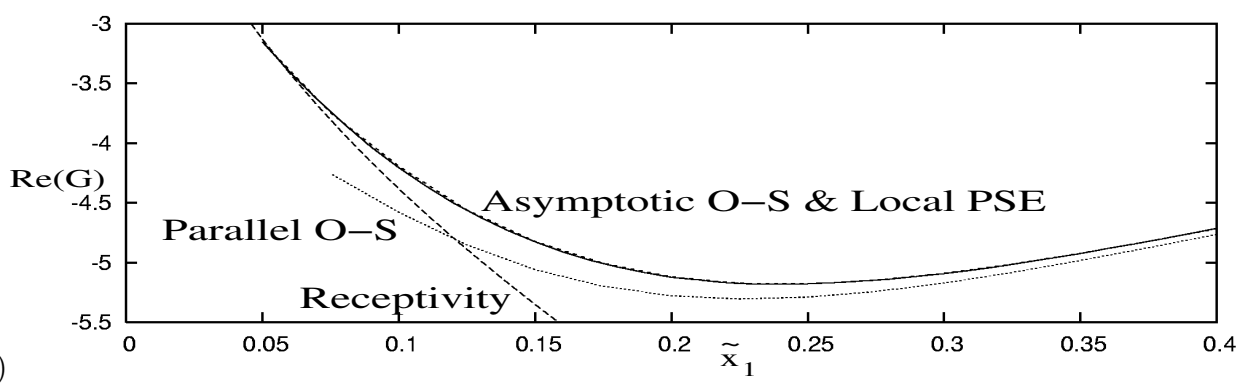

(b)

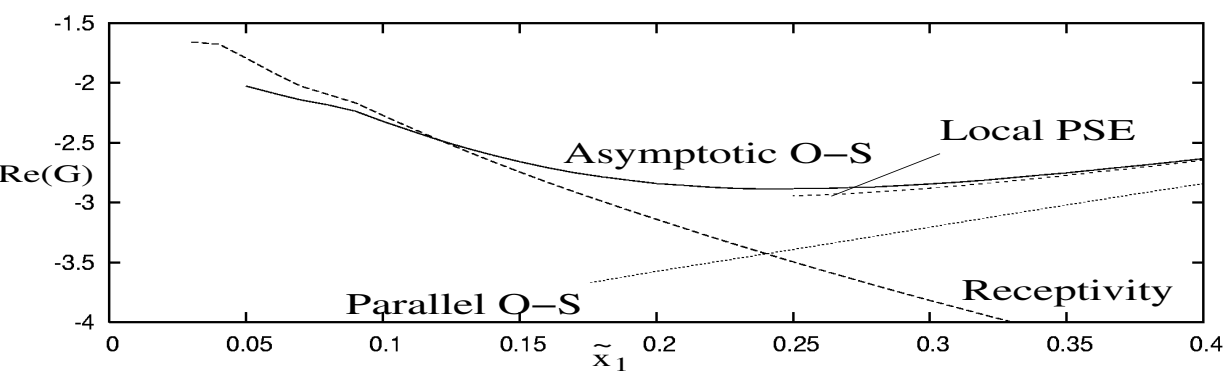

Figure 6. Plot of the real part of the growth rate, $G$, as a function of downstream distance, calculated by leading edge receptivity analysis, parallel Orr-Sommerfeld theory, local PSE theory, and asymptotic Orr-Sommerfeld theory for the cases (a) $\epsilon=0.05$ and (b) $\epsilon=0.1$.

the different methods described in the previous sections for two different values of $\epsilon$. The solid line shows the results for Goldstein's asymptotic results in the Orr-Sommerfeld region given by (3.5), up to and including the $O\left(\epsilon^{3}\right)$ term. This can be compared with results from parallel Orr-Sommerfeld theory, (4.1), and from local PSE, (4.4), which takes some account of non-parallel effects. For $\epsilon=0.05$ (figure 6(a)) results from asymptotic analysis and local PSE are indistinguishable, while the parallel flow results start to differ as the leading edge is approached, which is to be expected as non-parallel effects begin to dominate. As $\epsilon$ is increased to $\epsilon=0.1$ (figure 6(b)), the difference between the different solutions in the Orr-Sommerfeld region are larger. In addition, the local PSE solution can only be calculated for $\tilde{x}_{1} \gtrsim 0.25$ due to the first eigenvalue of (4.4) becoming indistinguishable from the other eigenvalues, as described earlier.

The existence of a matching region between the receptivity region close to the leading edge and the Orr-Sommerfeld region further downstream can also be seen in figure 6 . The dashed line marks the asymptotic growth rate of the first Lam-Rott mode given by (3.4). For the case $\epsilon=0.05$ (figure 6(a)), the receptivity results overlap the results from the asymptotic Orr-Sommerfeld and local PSE in the range $0.05<\tilde{x}_{1}<0.1$ and so a matching region clearly exists. For the larger value of $\epsilon$ (figure $6(\mathrm{~b})$ ), a reasonable match between the receptivity and asymptotic Orr-Sommerfeld results is seen at $\tilde{x}_{1} \approx 0.1$, but there is not such a clear matching region between the receptivity results and local PSE results due to the problem obtaining PSE results close enough to the leading edge.

Now that we have established the existence of the matching region, at least for sufficiently small $\epsilon$, we can try to utilise this result by starting our PSE analysis from inside or close to this region using (4.11) and (4.12) as the initial conditions. Figure 7 shows the real part of the growth rate, $G$, defined in (5.3), calculated using the PSE at different starting points, with the initial condition given by the receptivity analysis. Two starting positions were chosen, one lying within the matching region discussed above, and one further downstream where the LUBLE has become invalid. The results in figure 7 illustrate 
(a)

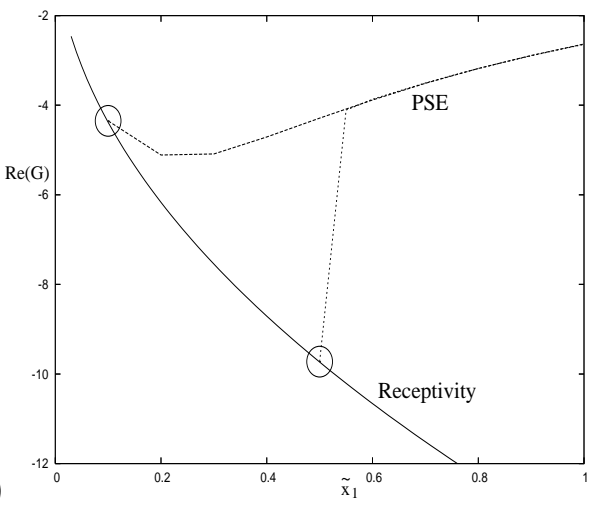

(b)

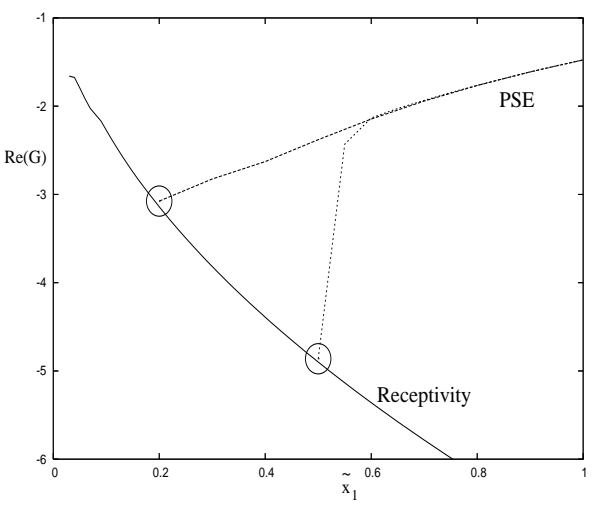

FIgURE 7. Plot of the real part of the growth rate, $G$, given by the PSE, started at different positions for (a) $\epsilon=0.05$ and (b) $\epsilon=0.1$.

the smallest possible value of $\tilde{x}_{1}^{(0)}$ at which the PSE could be started for the two values of $\epsilon$ considered, together with a sample calculation starting the PSE marching solution further downstream. When we attempted to use an initial condition further upstream of these smallest values, we found that the PSE would not iterate to the correct solution. The reason for this is discussed later in this section. We note that the minimum value of $\tilde{x}_{1}^{(0)}$ at which PSE marching solutions can be initiated increases as $\epsilon$ increases. The use of the initial condition further downstream highlights the fact that the PSE will iterate to the correct, even if an incorrect initial condition is imposed, as long as the point chosen is not too far downstream.

As well as the restriction that PSE calculations can not be started to close to the leading edge, there are other limitations in using the receptivity result as the initial condition. For example if we start with a receptivity boundary condition too far downstream, the PSE does not iterate to the correct solution, because the first initial jump in the eigenvalue is too large. However when a PSE run was started further downstream, with an initial eigenvalue taken from a previous calculation at that point, we found that the solution did indeed match onto the previous runs. This appears to suggest that the numerical scheme involved in the PSE needs a good initial approximation for the eigenvalue, but is less rigid when it comes to the initial mode shape, however this requires further investigation.

The use of the receptivity initial condition was also compared with PSE results using initial conditions from parallel Orr-Sommerfeld and local PSE analysis. For the case $\epsilon=0.1$, with $\tilde{x}_{1}^{(0)}=0.5$, the initial eigenvalues, $\alpha$, for the receptivity, Orr-Sommerfeld and local PSE analysis are given by $0.011416+0.011416 i, 0.012746+0.005918 i$ and $0.014008+$ $0.006338 i$ respectively, where $\alpha$ has been taken to be the most unstable eigenvalue in the last two cases. For this problem we chose a step size of $\Delta \tilde{x}_{1}=0.05$, and we found that the result of the PSE calculations with these initial conditions gave the same solution after the first 2 or 3 streamwise steps, which shows that our choice of a receptivity boundary condition is consistent with those previously used. In a similar fashion, it can be shown that using either of the pair of eigenvalues and corresponding mode shape from the local PSE analysis discussed in $\S 4.1$ leads to the same result downstream after 2 or 3 steps. Hence the ambiguity discussed in $\S 4.1$ is removed, and using either of the pair of initial eigenvalues is acceptable.

Another problem that arises if we try to start the PSE close to the leading edge is the appearance of transients due to the initial conditions. This is illustrated in figure 8 for the case $\epsilon=0.175\left(F=28.7 \times 10^{-6}\right)$, taking as the initial condition of the PSE code 
(a)
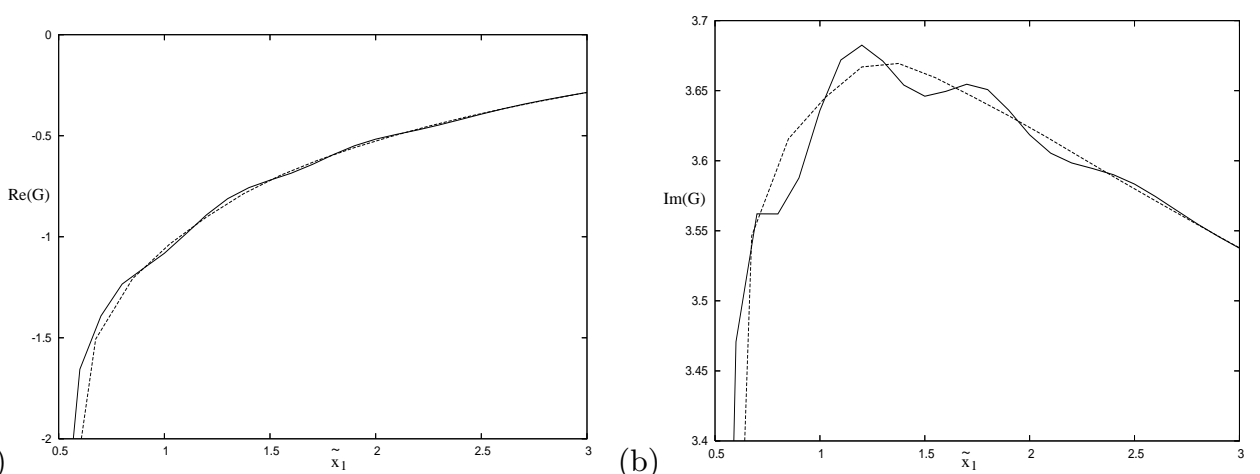

FIGURE 8. Plot of (a) the real part and (b) the imaginary part of $G$ as a function of downstream distance for $\epsilon=0.175$, showing the effects of transients from the initial conditions at $\tilde{x}_{1}=0.5$ for the step sizes 0.15 (solid line) and 0.175 (dotted line).

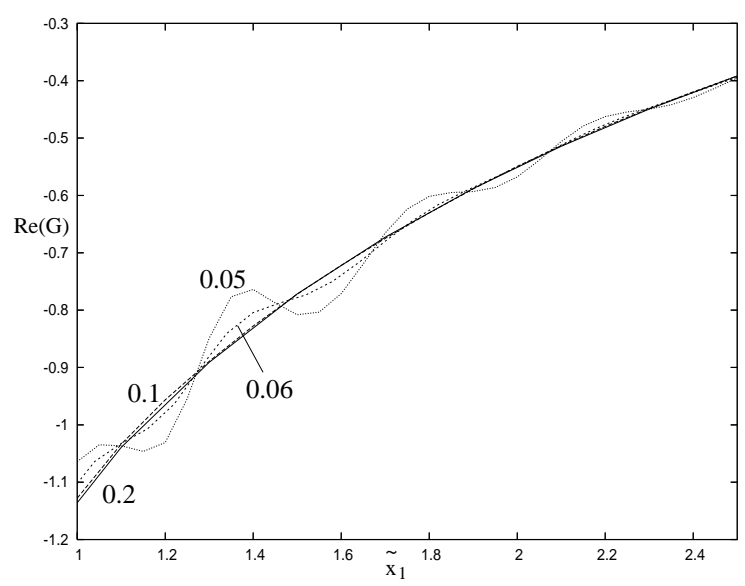

Figure 9. Plot of the initial transients on $\operatorname{Re}(G)$ for $\epsilon=0.15$ for 4 different step sizes, $\Delta \tilde{x}_{1}=0.05,0.06,0.1,0.2$, showing the occurrence of these transients as the step size reduces.

the receptivity result at $\tilde{x}_{1}=0.5$. With a step size of $\Delta \tilde{x}_{1}=0.15$ in the $\tilde{x}_{1}$ variable, oscillations of relative magnitude $15 \%$ appear in the range $0.5<\tilde{x}_{1}<2\left(6.3 \times 10^{4}<\right.$ $R_{x}<25.0 \times 10^{4}$ ), but these decay further downstream. Increasing the step size eliminates these oscillations. The presence of such transients has been noted by Bertolotti et al. (1992) and Herbert (1993), but there has been no systematic study of initial transients in PSE calculations. Understanding the origin of these transients is clearly important, though a detailed study is beyond the scope of the present paper. However some general observations can be made about the appearence of such transients. In figure 9 we see a more detailed plot of the transients on the real part of $G$ for the case $\epsilon=0.15$. We note that for the two largest step sizes, $\Delta \tilde{x}_{1}=0.2$ and 0.1 there are no oscillations, and the difference between these solutions is small. As we decrease the step size to $\Delta \tilde{x}_{1}=0.06$, we see these transients beginning to appear and as we decrease the step size further, the amplitude of these oscillations increases, while the wavelength remains approximately constant, $\lambda_{\tilde{x}_{1}} \approx 0.39$. One possible explanation for the appearance of these transients is that since the initial condition taken is only a numerical approximation to the first eigenmode, then the initial waveform is likely to contain a small contribution from higher eigenmodes. Initially these higher modes decay more slowly than the first eigenmode and 
hence these contributions may become significant. This is discussed in more detail in $\S 6$, but for the value of $\epsilon$ used in figure 9, it can be shown using Goldstein's asymptotics that the corresponding wavelength of the amplitude of the first eigenmode plus a small correction given by the second eigenmode is $\lambda_{\tilde{x}_{1}} \approx 0.36$. While this is not conclusive evidence of the origin of transients in PSE solutions, it is worthy of further investigation elsewhere. We believe it is a combination of these transients, which are related to the streamwise step size, as well as the difficulty finding the eigenvalue which leads to the failure of PSE convergence starting the calculation too close to the leading edge. The magnitude of the initial transient oscillations become increasingly large for smaller $\epsilon$ or smaller $\tilde{x}_{1}^{(0)}$, and in most cases become so large so quickly, that the PSE code fails to converge.

The results presented above, have focused on the initial (upstream) conditions suitable for PSE marching solutions. In particular it has been shown that conditions given by receptivity analysis in the region of the leading edge can be used as initial conditions. Combining receptivity analysis with stability calculations using numerical solutions of the PSE allows the determination of disturbance amplitudes at lower branch as a function of free-stream disturbance. We define the disturbance amplitude to be the absolute value of $\psi$ at the point where the real part of $\psi$ attains its maximum value, i.e where $\operatorname{Re}(\bar{\phi})=1$. We must take great care when evaluating the disturbance amplitude downstream, because of the

$$
\exp \left(\int^{x} G(x) d x\right)
$$

term in (5.2), which when we change variables to $\tilde{x}_{1}$ becomes

$$
\exp \left(\frac{U_{0}^{\prime 2}}{2 \epsilon^{2}} \int^{\tilde{x}_{1}} G\left(\tilde{x}_{1}\right) d \tilde{x}_{1}\right) .
$$

Any errors in the evaluation of the integral due to the step size $\Delta \tilde{x}_{1}$ are magnified for very small $\epsilon$. Thus we use Bode's rule for equally spaced mesh points, which has an error term of $O\left(\left(\Delta \tilde{x}_{1}\right)^{7}\right)$.

There are still two questions relating to the PSE which need addressing. Specifically, how is the disturbance amplitude downstream affected by changing the starting position of the PSE and by varying the step size? To address this, we define $\hat{A}\left(\tilde{x}_{1}^{(0)}, \tilde{x}_{1}\right)$ to be the disturbance amplitude at $\tilde{x}_{1}$, starting the PSE calculation at $\tilde{x}_{1}^{(0)}$, with the initial condition given by the receptivity result (4.11) and (4.12). Using this, the existence of a matching region between the receptivity results and the region over which PSE calculations are possible corresponds to the range of values of $\tilde{x}_{1}^{(0)}$ over which $\hat{A}$ is independent of $\tilde{x}_{1}^{(0)}$. Taking $\epsilon=0.05$ and a step size $\Delta \tilde{x}_{1}=0.05$, PSE calculations can not be started closer to the leading edge than $\tilde{x}_{1}^{(0)}=0.05$, for reasons explained earlier. Thus in figure 10 we plot the amplitude at $\tilde{x}_{1}=0.5$ as a function of starting position, but normalized by the value when $\tilde{x}_{1}^{(0)}=0.05$

$$
\tilde{A}\left(\tilde{x}_{1}^{(0)}\right)=\frac{\hat{A}\left(\tilde{x}_{1}^{(0)}, 0.5\right)}{\hat{A}(0.05,0.5)} .
$$

The position $\tilde{x}_{1}=0.5$ is chosen as the point of comparison of the amplitudes because it is far enough from the turning point in $\operatorname{Re}(G)$, that the change in growth rate is much smoother (see figure $7(\mathrm{a})$ ), thus not affecting any interpolation of the final point in the growth rate, which may introduce a small error. Taking larger values of $\tilde{x}_{1}$ at which to calculate the amplitude does not affect the results. For a PSE step-size of $\Delta \tilde{x}_{1}=0.05$, 


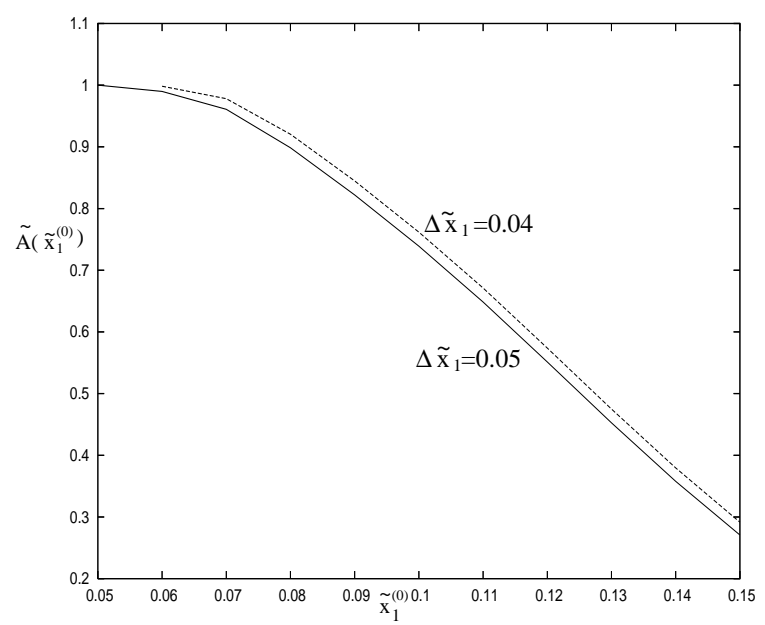

Figure 10. Plot of eigensolution amplitude at $\tilde{x}_{1}=0.5$ as a function of the starting point $\tilde{x}_{1}^{(0)}$ for $\epsilon=0.05$. The downstream amplitude is normalized with respect to the value given when $\tilde{x}_{1}^{(0)}=0.05$.

(a)

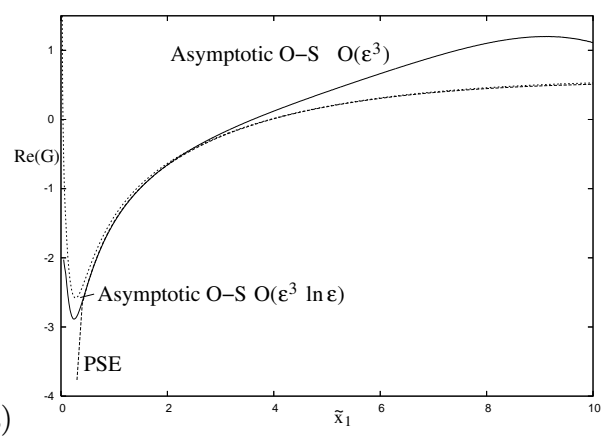

(b)

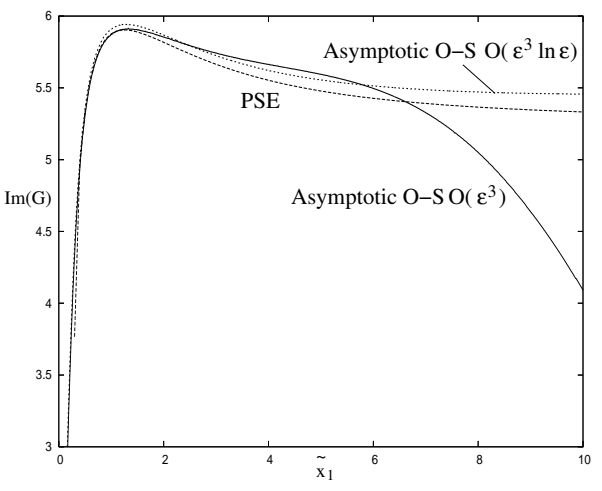

Figure 11. Plot of (a) the real part and (b) the imaginary part of $G$ as a function of downstream distance comparing the PSE result with that of the asymptotic Orr-Sommerfeld theory up to both $O\left(\epsilon^{3}\right)$ and $O\left(\epsilon^{3} \ln \epsilon\right)$ for $\epsilon=0.1$. Note $R_{x}=U_{\infty} x^{*} / \nu=U_{0}^{\prime 2} \tilde{x}_{1} /\left(2 \epsilon^{8}\right)$.

it is seen that for $0.05<\tilde{x}_{1}<0.1$ there is a $26 \%$ change in amplitude. This reinforces the earlier conclusion that a well defined matching region exists, at least for sufficiently small $\epsilon$. It is also apparent that changing the step size makes only a small change in the amplitude. Comparisons over a wider range of step sizes is not possible due to the appearence of transients as noted earlier.

Before we go on to compare these solutions with full scale numerics, we consider the comparison between growth rates obtained from PSE results and asymptotic OrrSommerfeld results downstream of the leading edge. In figure 11, we compare $G$ for $\epsilon=0.1$ calculated via the PSE and the asymptotic Orr-Sommerfeld calculations, up to and beyond the lower branch neutral stability point, which for this value of $\epsilon$ occurs at $\tilde{x}_{1}=3.946$. We show the asymptotic Orr-Sommerfeld solution calculated up to and including both $O\left(\epsilon^{3} \ln \epsilon\right)$ and $O\left(\epsilon^{3}\right)$ terms, to show the significant difference when the $O\left(\epsilon^{3}\right)$ term is added. We have very good agreement between the PSE and the $O\left(\epsilon^{3}\right)$ asymptotics up to the neutral stability point, but beyond this point the results differ. However after the neutral stability point we have much better agreement between the PSE and 
$O\left(\epsilon^{3} \ln \epsilon\right)$ asymptotics. Thus it appears that the $O\left(\epsilon^{3}\right)$ term becomes non-uniform with distance downstream.

The exact form of the $O\left(\epsilon^{3}\right)$ correction term is not considered here, but it contains complicated integrals, the limits of which depend on $\kappa$ (Goldstein 1982). The equation for $A_{\tilde{x}_{1}} / A$, which enters at order $\epsilon^{3}$, contains terms proportional to $\kappa$, which on inserting expansion (3.6) for $\kappa(\epsilon)$ leads to a solution in the form of an asymptotic expansion in powers of $\epsilon$. To consider a true asymptotic expansion, we require just the leading order term of $A\left(\tilde{x}_{1}\right) / A$, and hence use $\kappa_{0}$ in place of $\kappa$ in the original equation. However similar calculations using $\kappa$ up to and including $O\left(\epsilon^{3} \ln \epsilon\right)$ terms, and a full numerical value of $\kappa$ were considerably different from the results shown here, whereas the difference should only have been $O(\epsilon)$. This is due to shifting the contour in the complex plane over which integrals involving Airy functions are evaluated. The Airy functions oscillate rapidly, and decay, when their argument is between $-\pi / 3$ and $\pi / 3$, but away from the real axis, so a slight shift in the contour has a significant effect on the value of the integrals. Further analysis of the $O\left(\epsilon^{3}\right)$ term is of considerable interest but will not be considered here.

In summary, the results presented in this section show that this PSE method can be used to march the Tollmien-Schlichting wave disturbance downstream from the receptivity region to the neutral stability point, where we can calculate its amplitude in order to compare with other studies.

\section{Comparison with previous numerical studies}

In this paper we have described a method for obtaining the amplitude of the unstable component of the boundary layer disturbance at the lower branch neutral stability point and downstream of it in order to investigate the effect of free-stream forcing on transition. Attention is restricted to cases where the 'seeding' of unsteady disturbances in the boundary layer (known as receptivity) occurs in the region close to the leading edge. The actual form of free-stream forcing, be it due to acoustic wave or free-stream turbulence, only enters the analysis through a numerical receptivity coefficient, determined by asymptotic analysis of the leading edge region. For a flat plate it is possible to match asymptotic results from the receptivity region to asymptotic results from Orr-Sommerfeld theory and this was illustrated in $\S 4$. However the complexity of the Orr-Sommerfeld asymptotics for even a flat plate makes extension of these results to more general bodies impractical. Instead we choose to use results from the receptivity analysis as an initial condition for a numerical solution using the Parabolized Stability Equation. Figure 6 illustrates that for small $\epsilon$ an overlap region between the receptivity region and the Orr-Sommerfeld region exists which means that using receptivity results as an initial condition for PSE codes can be completely justified, though for larger values of $\epsilon$ more care is required.

To calculate the amplitude of the eigensolution at a given point downstream, we need to be able to integrate over the growth rate from some point in the receptivity region which we believe to be in the matching region, where the amplitude is known from (3.4) and the numerically determined value of the receptivity coefficient. This is straightforward for small values of $\epsilon$, as we can take the PSE right back to this matching region. However for larger $\epsilon$, we have to 'patch' this region using a curve fitting technique, because we have to start our PSE calculation downstream of the matching region. We patch the function in the range $\tilde{x}_{\alpha}<\tilde{x}_{1}<\tilde{x}_{\beta}$, where $\tilde{x}_{\alpha}$ is a point in the receptivity region that we believe to be in the matching region, and $\tilde{x}_{\beta}$ is the closest point to the leading edge that we could get the result in the Orr-Sommerfeld region. We require that the patching 
(a)

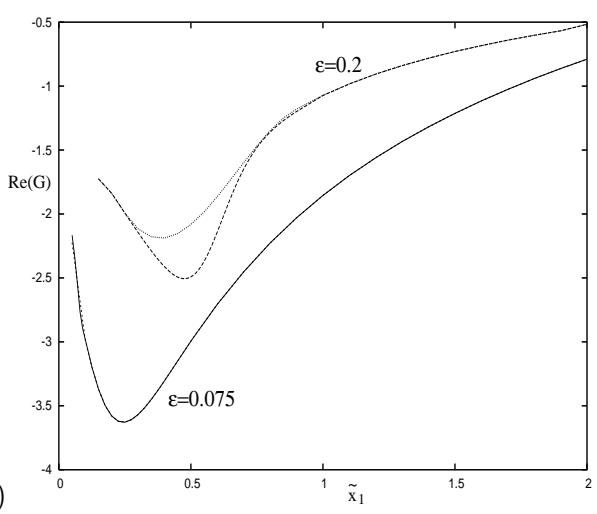

(b)

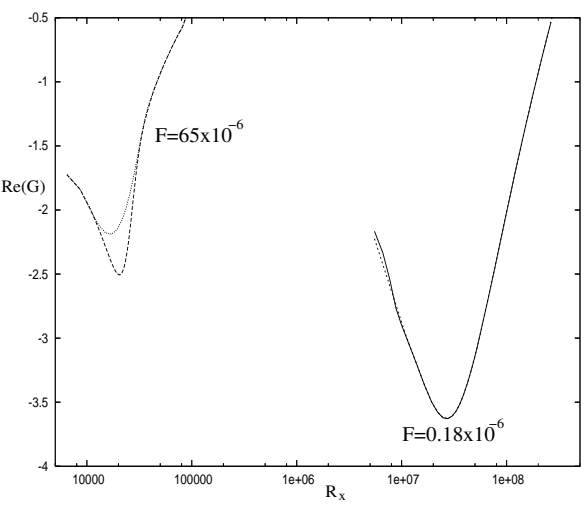

Figure 12. Plot of growth rates, $\operatorname{Re}\left(G_{1}\right)$ and $\operatorname{Re}\left(G_{2}\right)$, for $\epsilon=0.075\left(F=0.18 \times 10^{-6}\right)$ which requires very minimal patching, and $\epsilon=0.2\left(F=65 \times 10^{-6}\right)$ which requires much more patching, plotted as (a) a function of $\tilde{x}_{1}$ and (b) a function of $R_{x}=U_{\infty} x^{*} / \nu=U_{0}^{\prime 2} \tilde{x}_{1} /\left(2 \epsilon^{8}\right)$. The lower of the two curves for the $\epsilon=0.2$ case corresponds to $\operatorname{Re}\left(G_{1}\right)$.

function satisfies

$$
G\left(\tilde{x}_{1}\right) \approx\left\{\begin{array}{ll}
f_{1}\left(\tilde{x}_{1}\right) & \tilde{x}_{1}<\tilde{x}_{\alpha} \\
f_{2}\left(\tilde{x}_{1}\right) & \tilde{x}_{1}>\tilde{x}_{\beta}
\end{array},\right.
$$

or better still equality, where the function $f_{1}$ is the asymptotic receptivity result and $f_{2}$ is the PSE result with the first few iterations removed so that we can be sure we are on the growth rate curve. We also use the local PSE to extend the PSE results upstream closer to the leading edge to make patching easier.

We consider two methods to patch the region between $\tilde{x}_{\alpha}$ and $\tilde{x}_{\beta}$. For the first one we define $G_{1}$ to be

$$
G_{1}\left(\tilde{x}_{1}\right)=\lambda_{1}\left(\tilde{x}_{1}\right) \tilde{f}_{1}\left(\tilde{x}_{1}\right)+\lambda_{2}\left(\tilde{x}_{1}\right) \tilde{f}_{2}\left(\tilde{x}_{1}\right)
$$

where

and

$$
\lambda_{1}=\frac{1}{2}(1-\tanh \theta) \text { and } \lambda_{2}=\frac{1}{2}(1+\tanh \theta)
$$

$$
\theta=\frac{5\left(\tilde{x}_{1}-\frac{1}{2}\left(\tilde{x}_{\alpha}+\tilde{x}_{\beta}\right)\right)}{\tilde{x}_{\beta}-\tilde{x}_{\alpha}} .
$$

The function $\tilde{f}_{1}$ is taken to be the straight line extension of $f_{1}$ from $\tilde{x}_{\alpha}$ to $\tilde{x}_{\beta}$, and $\tilde{f}_{2}$ is taken to be the straight line extension of $f_{2}$ from $\tilde{x}_{\beta}$ to $\tilde{x}_{\alpha}$. For the second, we define $G_{2}$ to be

$$
G_{2}\left(\tilde{x}_{1}\right)= \begin{cases}f_{1}\left(\tilde{x}_{1}\right) & \tilde{x}_{1}<\tilde{x}_{\alpha}, \\ A \tilde{x}_{1}^{3}+B \tilde{x}_{1}^{2}+C \tilde{x}_{1}+D & \tilde{x}_{\alpha}<\tilde{x}_{1}<\tilde{x}_{\beta}, \\ f_{2}\left(\tilde{x}_{1}\right) & \tilde{x}_{1}>\tilde{x}_{\beta},\end{cases}
$$

where $A, B, C$ and $D$ are constants chosen so that $G_{2}$ and its derivative are continuous at $\tilde{x}_{\alpha}$ and $\tilde{x}_{\beta}$.

The results of the patching on the growth rates, $\operatorname{Re}\left(G_{1}\right)$ and $\operatorname{Re}\left(G_{2}\right)$, can be seen for two values of $\epsilon$ in figure 12. For the case $\epsilon=0.075$, only a small amount of patching was required around $\tilde{x}_{1}=0.1$ and both methods gave similar results, leading to an $8 \%$ difference in the T-S wave amplitude at lower branch. However when $\epsilon=0.2$, we had to patch a much larger region between $0.25<\tilde{x}_{1}<1.0$, which leads to the growth rate curve of $G_{1}$ possibly dropping more rapidly between $0.5<\tilde{x}_{1}<1.0$ than expected when we compare its shape to the $\epsilon=0.075$ curve. This gives a $22 \%$ difference in T-S 
(a)

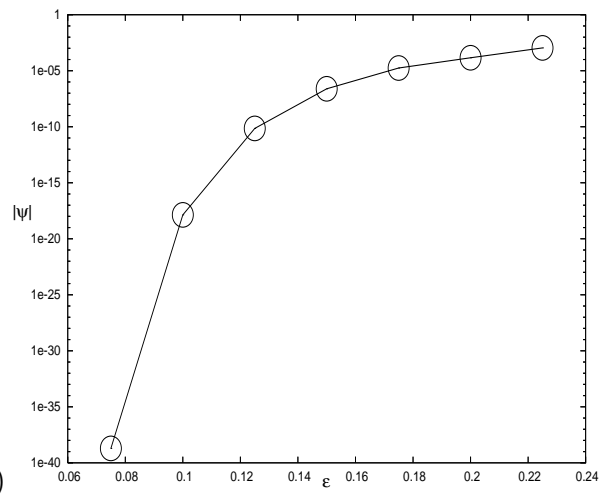

(b)

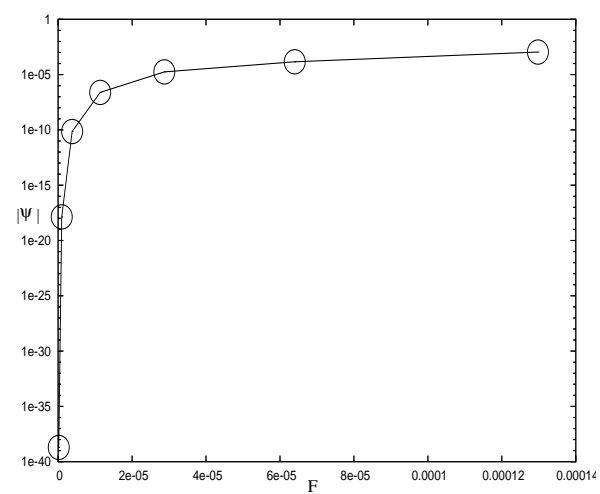

FIgURE 13. Plot of eigensolution amplitude at the lower branch neutral stability point as (a) a function of $\epsilon$ and (b) a function of $F=\epsilon^{6}$.

wave amplitudes at lower branch, for the two patching methods. However the $G_{2}$ curve appears to give a shape similar to the $\epsilon=0.075$ curve, and this curve also gives better agreement with Goldstein's asymptotics. It is because of this reason that we choose to use this patching technique for the remainder of the paper.

Using our chosen method, the amplitude of the T-S wave can be calculated at all points downstream. Figure 13 shows the amplitude of the T-S wave at the lower branch neutral stability point as a function of $\epsilon$ where the free-stream disturbance is taken to be an acoustic wave propagation parallel to the mean flow. In this case, the receptivity coefficient in (4.12) is given by Goldstein et al. (1983) as $\left|C_{1}\right|=0.9662$. Results for very small $\epsilon$ are asymptotically valid due to the well-defined matching region, while results for larger values of $\epsilon$ involve numerical patching but do allow comparison with experimental and numerical results.

Haddad \& Corke (1998) consider a parabola at zero angle of incidence to a uniform flow with a small amplitude acoustic disturbance propagating parallel to the mean flow. The steady flow around the body is solved numerically and the unsteady disturbance obtained by solving a linear perturbation. Downstream, the unsteady disturbance consists of a Stokes-wave determined by the local forcing at that location, together with a sum of Tollmien-Schlichting waves. Upstream of the first neutral stability point, the T-S waves are small compared with the Stokes wave. The asymptotic form of the Stokes wave far downstream can be obtained (Hammerton 1999), but instead Haddad \& Corke obtain a numerical approximation to the Stokes solution by solving the unsteady equation with convective-inertia terms dropped. If we form an asymptotic solution for the Stokes solution used by Haddad \& Corke, in powers of $\epsilon=R e^{-\frac{1}{6}}$, we find

$$
\psi_{S T}=(2 \xi)^{\frac{1}{2}} \eta+O\left(\epsilon^{6}\right),
$$

at the outer edge of the boundary layer. However Hammerton (1999) found the same solution to be

$$
\psi_{S T}=(2 \xi)^{\frac{1}{2}}\left(\eta+O\left(\xi^{-\frac{1}{2}}\right)\right)+O\left(\epsilon^{6}\right),
$$

where the $O\left(\xi^{-\frac{1}{2}}\right)$ correction terms come from the inclusion of the convective-inertia terms. Having obtained an expression for the Stokes solution, this is subtracted from the unsteady solution in order to provide an approximation to the magnitude of the T-S waves, after a filtering process in which any waves of wavelength greater than $2 \lambda_{T S}$ are removed. This process should remove any remaining contribution by the Stokes solution. Haddad \& Corke check their method against existing asymptotics on a flat plate by 


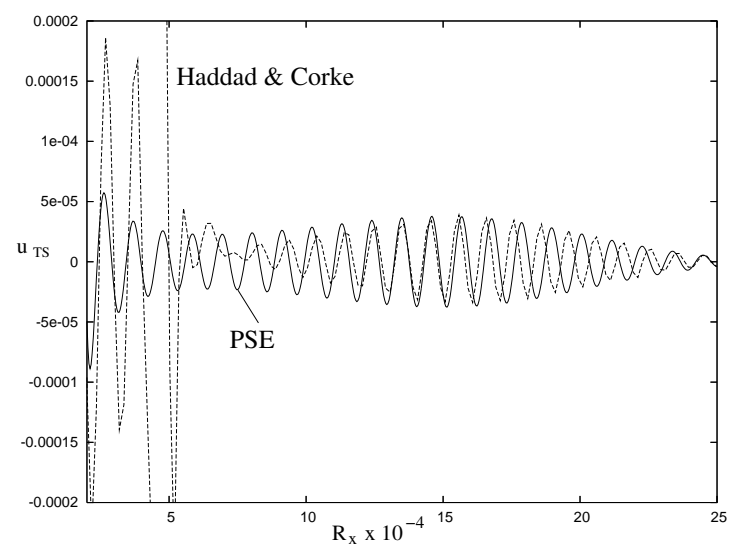

Figure 14. Plot of the T-S wave velocity, $u_{T S}$, as a function of $R_{x}=U_{\infty} x^{*} / \nu$ at the level $\eta=0.033$, for both Haddad \& Corke's method, and our PSE method, for $\epsilon=0.248$ $\left(F=230 \times 10^{-6}\right)$.

considering the limit as the nose radius goes to zero, and it is against these results, that we check our numerical scheme.

Figure 14 shows a plot of the streamwise velocity, $u_{T S}$, at $\eta=0.033$ as a function of $R_{x}=U_{\infty} x^{*} / \nu$, where $x^{*}$ is a dimensional distance from the leading edge. The results of the present paper (solid line) agree well with the results of Haddad \& Corke (dotted line) (cf figure 13(b) from Haddad \& Corke (1998), after the data has been filtered), downstream of the lower branch point and in particular around the upper branch of the neutral stability curve. The discrepancy between the two sets of results around the lower branch point could be down to two factors. The value of $\epsilon$ is relatively large and hence we are considering points close to the turning point of the neutral stability curve where all numerical methods are very sensitive (see Schmid \& Henningson (2001), figure 7.30). In addition, any numerical errors associated with the subtraction of the Stokes wave and the filtering of higher modes in Haddad \& Corke are likely to be most significant at this point since the unstable T-S wave has its lowest amplitude there. Comparison with experiments is difficult for the flat plate, however our comparisons with Haddad \& Corke's numerics strongly suggests that our receptivity/PSE method is valid.

Saric \& Rasmussen (1992) conducted experiments on a flat plate with an elliptical leading edge stuck onto it, but it was noted that the discontinuity in curvature, at the join, produced another region of receptivity. Saric et al. (1995) eliminated this problem by using an elliptic leading edge machine drilled onto a flat plate, called a Modified Super Ellipse (MSE), to eliminate any discontinuity, and further experiments were carried out by Saric \& White (1998). Fuciarelli et al. (1998) and Wanderley \& Corke (2001) both computed their respective numerical calculations for the MSE in order to compare their results with those of Saric \& White. Excellent agreement of the T-S wave amplitude at lower branch is found between Wanderley \& Corke and the experiments, however in this paper we are not able to make comparisons with these results, due to the significance of non-zero pressure gradients along a MSE.

As well as producing amplitude results at lower branch to compare with experimental measurements, the numerical methods of Corke and co-workers were used to compare with leading edge receptivity results. Wanderley \& Corke define a general form of the receptivity coefficient as the ratio of the maximum T-S wave amplitude at an $x$-location to the amplitude of the free-stream disturbance, and denote it by $K_{x}=\left|\left(u_{T S}\right)\right| /\left|\left(u_{\infty}\right)\right|$, 


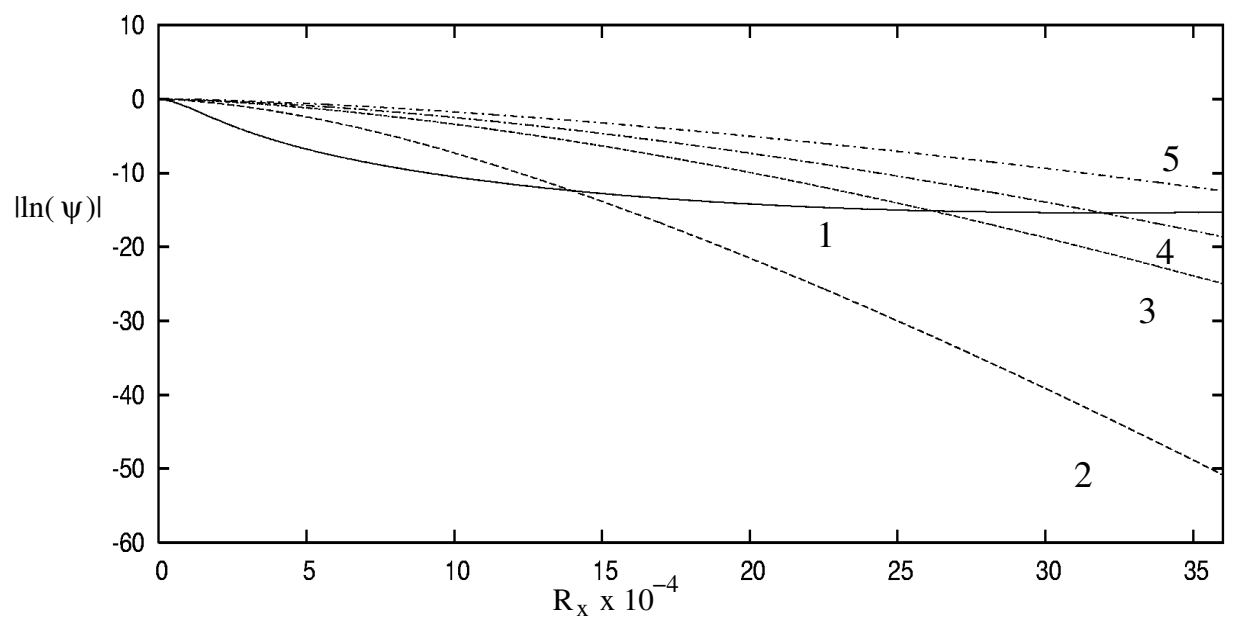

Figure 15. Plot of the $\log$ of the amplitudes $\left(\int \operatorname{Re}(G) d x_{R}\right)$ of the first 5 T-S modes as a function of $R_{x}$, with $\epsilon=0.194\left(F=54.0 \times 10^{-6}\right)$.

where $u_{T S}=\partial \psi / \partial y$ is the T-S wave after filtering and $u_{\infty}$ is the free-stream disturbance. This definition of the receptivity coefficient depends on downstream distance and has a very different meaning than the receptivity coefficient defined in asymptotic analysis.

By considering results close to the neutral stability point, Corke and co-workers assumed that the $1^{s t} \mathrm{~T}-\mathrm{S}$ wave dominates the solution, and extrapolate the amplitude of this wave back to the leading edge in order to compare their numerical results with the receptivity results of Goldstein (1983) and Hammerton \& Kerschen (1996). For the MSE, Wanderley \& Corke considered the disturbance amplitude in a region just upstream of the lower branch neutral stability point believing that in this region the first T-S mode dominates. We are able to investigate this assumption in figure 15 , which shows the $\log$ of the amplitudes $\left(\ln (\psi)=\int \operatorname{Re}(G) d x_{R}\right)$ of the first 5 eigenfunctions calculated using Goldstein's asymptotic method as a function of the streamwise Reynolds number, $R_{x}$, where the neutral stability point occurs at $R_{x} \approx 3.1 \times 10^{5}$. The corresponding Reynolds number based on the boundary layer thickness can be calculated using $R_{\delta}=1.72 R_{x}^{\frac{1}{2}}$, and in this variable the neutral stability point occurs at $R_{\delta}=958$. In the region $2 \times 10^{5}<R_{x}<3 \times 10^{5}\left(769<R_{\delta}<942\right)$ considered by Wanderley \& Corke it does not appear that the $1^{\text {st }}$ T-S mode dominates the $3^{\text {rd }}, 4^{\text {th }}$ and $5^{\text {th }}$ T-S modes, although it does dominate the $2^{\text {nd }}$. However Goldstein et al. (1983) showed that for a flat plate the receptivity coefficients multiplying the $3^{\text {rd }}, 4^{\text {th }}$ and $5^{\text {th }}$ T-S modes, are at least 5 times smaller than the coefficient multiplying $1^{\text {st }}$ T-S mode. Hence if similar results hold for the rounded leading edge geometry considered, then the assumption that the first T-S mode dominates all other T-S modes may indeed be valid.

Wanderley \& Corke then assume that this T-S mode has constant decay rate at all locations back to the leading edge and an amplitude of the unsteady disturbance is found at the leading edge, though the physical interpretation of such a quantity is unclear. This extrapolation is marked as the dotted line in figure 16 ( $\mathrm{cf}$ figure 10 of Wanderley \& Corke (2001)) for $\epsilon=0.194\left(F=54.0 \times 10^{-6}\right)$. However this analysis does have some possible flaws. The most important of these is the assumption of constant decay rate between the leading edge and the lower branch neutral stability point. If the extrapolation was performed on results closer to the neutral stability point the measured decay rate would be much less and the value of $K_{x}$ extrapolated to the leading edge would be much smaller. 


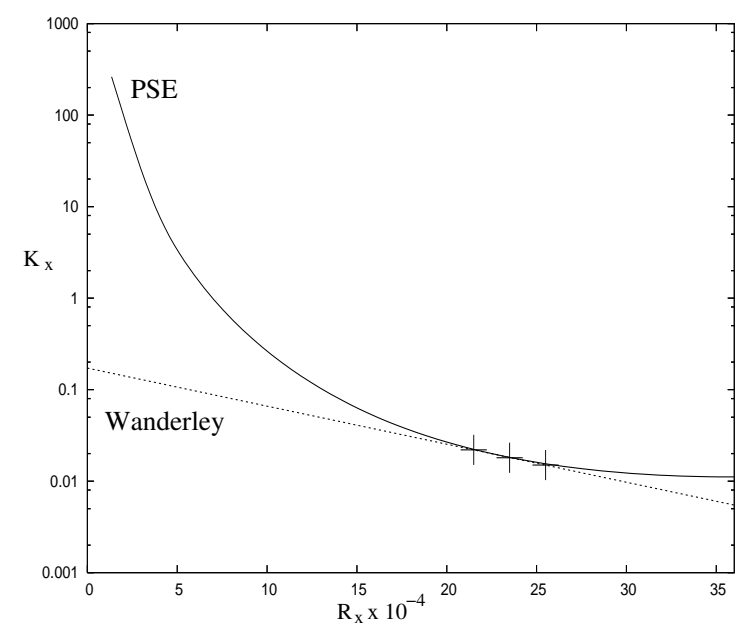

Figure 16. Plot of figure 10 from Wanderley \& Corke (2001) for a 20:1 MSE with the results from PSE calculations for $\epsilon=0.194\left(F=54.0 \times 10^{-6}\right)$.

Results of this analysis for a flat plate are not available, but in figure 16 the results of the present paper for a flat plate are compared with the numerical results of Wanderley \& Corke for a Modified Super Ellipse. This shows that for a flat plate the assumption of constant growth rate is not valid in this case. It is possible that the points calculated by Wanderley \& Corke in figure 16 could be solely that of the $1^{\text {st }} \mathrm{T}-\mathrm{S}$ mode, for the reasons discussed earlier. However a better comparison between leading edge receptivity analysis and the numerical analysis of Wanderley \& Corke would be possible if T-S amplitudes slightly downstream of the lower branch neutral stability point were available, since then there would be no question that the unstable T-S mode dominated the solution as seen in figure 14. Using results from PSE calculations for the particular geometry would then allow the extrapolation of the T-S amplitude to positions closer to the leading edge. This would then allow comparison with the receptivity coefficients used in asymptotic investigations which have more physical relevance in this leading edge region than the $K_{L E}$ calculated by Wanderley \& Corke.

\section{Conclusions}

To conclude, the method presented in this paper has demonstrated that for a flat plate we can accurately calculate T-S mode amplitudes at the lower branch neutral stability point for sufficiently small $\epsilon$ (large $R e$ ) where a well defined matching region is apparent. We also demonstrated that for larger $\epsilon$ we can patch the data from the PSE to that of the leading edge asymptotics and produce T-S wave calculations which agree well with numerical data. Adjoint methods (Hill 1995) have recently been extended to look at the receptivity problem (Giannetti 2002), and this is a future area of comparison. The present PSE method can be readily extended to more general geometries. Using this method for the Modified Super Ellipse would allow us to investigate the importance of leading edge curvature on T-S wave amplitudes and allow better comparison with the numerical results obtained by Wanderley \& Corke as well as experimental data. In addition, quantitative comparison between results of asymptotic receptivity analysis for different free-stream disturbances and existing experiments will be possible. This is work currently in progress. 
The authors would like to thank the referees for their interesting and useful comments towards this paper.

\section{REFERENCES}

Andersson, P., Henningson, D. S. \& Hanifi, A. 1998 On a stabilization procedure for the parabolic stability equations. J. Engrg. Math. 33 (3), 311-332.

Bertolotti, F. P., Herbert, T. \& Spalart, P. R. 1992 Linear and nonlinear stability of the Blasius boundary layer. J. Fluid Mech 242, 441-474.

Bodonyi, R. J., Welch, J. C., Duck, P. W. \& Tadjfar, M. 1989 A numerical study of the interaction between unsteady free-stream disturbances and localized variations in surface geometry. J. Fluid Mech. 209, 285-308.

Bridges, T. J. \& Morris, P. J. 1984 Differential eigenvalue problems in which the parameter appears nonlinearly. J. Comput. Phys. 55 (3), 437-460.

Brown, S. N. \& Stewartson, K. 1973 On the propagation of disturbances in a laminar boundary layer. Proc. Camb. phil. Soc 73, 493-514.

Choudhari, M. \& Streett, C. 1992 A finite Reynolds number approach for the prediction of boundary-layer receptivity in localized regions. Phys. Fluids A 4:2, 495-514.

Crouch, J. D. 1992 Non-localized receptivity of boundary layers. J. Fluid Mech. 244, 567-581.

Erturk, E. \& Corke, T. C. 2001 Boundary layer receptivity to sound at incident angles. $J$. Fluid Mech. 444, 383-407.

Fuciarelli, D. A., Reed, H. L. \& Lyttle, I. 1998 DNS of leading-edge receptivity to sound. AIAA Paper 98-2644.

Gaster, M. 1974 On the effects of boundary-layer growth on flow stability. J. Fluid Mech. 66, $465-480$.

Giannetti, F. 2002 Boundary Layer Receptivity. PhD Thesis, University of Cambridge, Cambridge, United Kingdom.

Goldstein, M. E. 1982 Generation of Tollmien-Schlichting waves by free-stream disturbances at low Mach numbers. NASA TM 83026.

Goldstein, M. E. 1983 The evolution of Tollmien-Schlichting waves near a leading edge. $J$. Fluid Mech. 127, 59-81.

Goldstein, M. E. 1985 Scattering of acoustic waves into Tollmien-Schlichting waves by small streamwise variations in surface geometry. J. Fluid Mech. 154, 509-529.

Goldstein, M. E. \& Hultgren, L. S. 1987 A note on the generation of Tollmien-Schlichting waves by sudden surface-curvature change. J. Fluid Mech. 181, 519-525.

Goldstein, M. E., Leib, S. J. \& Cowley, S. J. 1992 Distortion of a flat-plate boundary layer by free-stream vorticity normal to the plate. J. Fluid Mech. 237, 231-260.

Goldstein, M. E., Sockol, P. M. \& Sanz, J. 1983 The evolution of Tollmien-Schlichting waves near a leading edge. Part 2. Numerical determination of amplitudes. J. Fluid Mech. 129, 443-453.

Haddad, O. M. \& CoRke, T. C. 1998 Boundary layer receptivity to free-stream sound on parabolic bodies. J. Fluid Mech. 368, 1-26.

Haddad, O. M., Erturk, E. \& Corke, T. C. 2005 Acoustic receptivity of the boundary layer over parabolic bodies at angles of attack. J. Fluid Mech. 536, 377-400.

Hammerton, P. W. 1999 Comparison of Lam-Rott and Brown-Stewartson eigensolutions of the boundary-layer equations. Quart. J. Mech. Appl. Math. 52 (3), 373-385.

Hammerton, P. W. \& Kerschen, E. J. 1996 Boundary-layer receptivity for a parabolic leading edge. J. Fluid Mech. 310, 243-267.

Hammerton, P. W. \& Kerschen, E. J. 2005 Leading-edge receptivity for bodies with mean aerodynamic loading. J. Fluid Mech. 535, 1-32.

HeinRICh, R. \& Kerschen, E. J. 1989 Leading-edge boundary-layer receptivity to free-stream disturbance structures. ZAMM 69:T596 .

Herbert, T. 1993 Parabolized Stability Equations. AGARD Rep., 4-1 to 4-34 .

HiLl, D. C. 1995 Adjoint systems and their role in the receptivity problem for boundary layers. J. Fluid Mech. 292, 183-204.

Kerschen, E. J., Choudhari, M. \& Heinrich, R. A. 1990 Generation of boundary instability 
waves by acoustic and vortical freestream disturbances. Laminar-Turbulent Transition, Vol III. New York:Springer pp. 477-488.

Lam, S. H. \& RotT, N. 1960 Theory of linearized time-dependent boundary layers. Cornell University GSAE Rep. AFOSR pp. TN-60-1100.

Morkovin, M. V. 1969 On the many faces of transition. In Viscous Drag Reduction, ed. C. S. Wells. New York: Plenum.

Morkovin, M. V. 1985 Guide to experiments on instability and laminar-turbulent transition in shear layers. Cincinnati, Ohio.

ReEd, H. L. 1994 Direct numerical simulation of transition: the spatial approach. Progress in Transition Modelling, AGARD report 793. Paris: NATO, 6.1-46 .

SARIC, W. \& NAYFeH, A. 1975 Nonparallel stability of boundary-layer flows. Phys. Fluids 18, 945-950.

Saric, W. S. \& Rasmussen, B. K. 1992 Boundary layer receptivity: Freestream sound on an elliptic leading edge. Bull. Am. Phys. Soc. 37, 1720.

Saric, W. S., Reed, H. L. \& Kerschen, E. J. 1994 Leading edge receptivity to sound: experiments, DNS, and theory. AIAA Paper 94-2222 .

SARIC, W. S., ReEd, H. L. \& Kerschen, E. J. 2002 Boundary-layer receptivity to freestream disturbances. Annu. Rev. Fluid Mech. 34, 291-319.

SARIC, W. S., Wei, W. \& RAsmussen, B. K. 1995 Effect of leading-edge on sound receptivity. Laminar-Turbulent Transition, (IUTAM Sendai, 1994, ed. R. Kobayashi), Springer, 413420 .

Saric, W. S. \& White, E. B. 1998 Influence of high-amplitude noise on boundary-layer transition to turbulence. AIAA Paper 98-2645 .

Schmid, P. J. \& Henningson, D. S. 2001 Stability and Transition in Shear Flows. Springer .

Wanderley, J. B. V. \& Corke, T. C. 2001 Boundary layer receptivity to free-stream sound on elliptic edges of flat plates. J. Fluid Mech. 429, 1-21. 